\title{
An indecomposable and unconditionally saturated Banach space
}

\author{
by \\ Spiros A. Argyros (Athens) and Antonis Manoussakis (Chania) \\ Dedicated to Aleksander Petczyński \\ on the occasion of his 70th birthday
}

\begin{abstract}
We construct an indecomposable reflexive Banach space $X_{\text {ius }}$ such that every infinite-dimensional closed subspace contains an unconditional basic sequence. We also show that every operator $T \in \mathcal{B}\left(X_{\text {ius }}\right)$ is of the form $\lambda I+S$ with $S$ a strictly singular operator.
\end{abstract}

1. Introduction. The aim of this paper is to present a Banach space which is not the sum of two infinite-dimensional closed subspaces $Y, Z$ with $Y \cap Z=\{0\}$ and which has the property every closed subspace of it contains an unconditional basic sequence. We shall denote this space by $X_{\text {ius }}$. W. T. Gowers' famous dichotomy, [G3], provides an alternative description of this space. Namely $X_{\text {ius }}$ is an indecomposable Banach space with no hereditarily indecomposable (H.I.) subspace. The problem of the existence of such spaces was posed by H. P. Rosenthal and it is stated in [G2]. The interest for such spaces arises from the coexistence of conditional (indecomposable) and unconditional (unconditionally saturated) structure. This is a free translation of W. T. Gowers' comments preceding the statement of the problem of the existence of such spaces in [G2] (Problem 5.11). We should mention that indecomposable spaces which are not H.I. are already known. For example, $[\mathrm{AF}]$ provides reflexive H.I. spaces $X$ such that $X^{*}$ contains an unconditional basic sequence. The methods used in $[\mathrm{AF}]$ do not seem to be capable of providing H.I. spaces $X$ with $X^{*}$ unconditionally saturated.

The space presented in this paper is built following ideas used for the construction of H.I. Banach spaces. Our method is an adaptation of [AD] constructions as extended in [AT1]. Both are variations of the fundamental

2000 Mathematics Subject Classification: Primary 46B20.

Key words and phrases: indecomposable Banach space, unconditionally saturated, reflexive Banach space. 
discovery of W. T. Gowers and B. Maurey $[\mathrm{GM}]$. In our case we use as an unconditional frame a mixed Tsirelson space $T\left[\left(\mathcal{A}_{n_{j}}, 1 / m_{j}\right)_{j}\right]$ which has similar properties to Th. Schlumprecht's space $S$ (see $[\mathrm{S}]$ ). The norming set $K$ of the space $X_{\text {ius }}$ is a subset of the unit ball of the dual of $T\left[\left(\mathcal{A}_{n_{j}}, 1 / m_{j}\right)_{j}\right]$. The only feature in which the space $X_{\text {ius }}$ differs from the corresponding construction of a H.I. space concerns the definition of the special functionals. The key observation that changing the special functionals one could obtain interesting non-H.I. spaces is due to W. T. Gowers and it was used for the solution of important and long standing problems in the theory of Banach spaces $[\mathrm{G}]$.

For the space $X_{\text {ius }}$ we need the special functionals to be defined in such a way that the following geometric property holds. For every $Y=\left\langle e_{n}\right\rangle_{n \in M}$, where $M \in\left[\mathbb{N}\right.$ ] (i.e. $M$ is an infinite subset of $\mathbb{N}$ ) and $\left(e_{n}\right)_{n \in \mathbb{N}}$ is the natural basis of $X_{\text {ius }}$, the quotient map $Q: X_{\text {ius }} \rightarrow X_{\text {ius }} / Y$ is strictly singular. This is equivalent to saying that $\operatorname{dist}\left(S_{Z}, S_{Y}\right)=0$ for every infinitedimensional subspace $Z$ of $X_{\text {ius }}$. This property clearly holds in the case of H.I. spaces. In our case we define the special functionals in such a way that the aforementioned property holds; on the other hand we attempt to keep the dependence inside each special functional as small as possible. Thus if we go deeper into the structure of any subspace of $X_{\text {ius }}$ the action of the special functionals becomes negligible, which permits us to find unconditional basic sequences. Another property of $X_{\text {ius }}$ concerns bounded linear operators. Namely every $T: X_{\text {ius }} \rightarrow X_{\text {ius }}$ is of the form $T=\lambda I+S$, where $S$ is strictly singular. Thus $X_{\text {ius }}$ is not isomorphic to any of its proper subspaces.

After submitting the present paper for publication A. Tolias and the first author provided a dual pair $X, X^{*}$ of separable reflexive Banach spaces such that $X$ is unconditionally saturated and $X^{*}$ is H.I. (see [AT2]). The construction of this dual pair makes use of the results and techniques of the present paper.

2. Definition of the space $X_{\text {ius }}$. We shall use the standard notation. Thus $c_{00}$ denotes the linear space of all eventually zero sequences, and for $x \in c_{00}$ we write $\operatorname{supp} x=\{n: x(n) \neq 0\}$ and denote by range $(x)$ the minimal interval of $\mathbb{N}$ containing $\operatorname{supp} x$. Also for $x, y \in c_{00}$ by writing $x<y$ we mean that $\max \operatorname{supp} x<\min \operatorname{supp} y$. We shall also use the standard results from the theory of bases of Banach spaces as described in [LT].

We choose two strictly increasing sequences $\left(n_{j}\right)_{j},\left(m_{j}\right)_{j}$ of positive integers such that

(i) $m_{1}=2$ and $m_{j+1}=m_{j}^{5}$,

(ii) $n_{1}=4$ and $n_{j+1}=\left(4 n_{j}\right)^{s_{j}}$, where $2^{s_{j}} \geq m_{j+1}^{3}$. 
Let $\mathbf{Q}$ be the set of scalar sequences with finite nonempty support, rational coordinates and maximum at most 1 in modulus. We also set

$$
\begin{aligned}
\mathbf{Q}_{s}=\left\{\left(x_{1}, f_{1}, \ldots, x_{n}, f_{n}\right): x_{i}, f_{i} \in \mathbf{Q}, i=1, \ldots, n,\right. \\
\left.\quad \operatorname{range}\left(x_{i}\right) \cup \operatorname{range}\left(f_{i}\right)<\operatorname{range}\left(x_{i+1}\right) \cup \operatorname{range}\left(f_{i+1}\right) \forall i<n\right\} .
\end{aligned}
$$

We consider a coding function $\sigma$ (i.e. an injection) from $\mathbf{Q}_{s}$ to the set $\{2 j$ : $j \in \mathbb{N}\}$ such that for every $\phi=\left(x_{1}, f_{1}, \ldots, x_{n}, f_{n}\right) \in \mathbf{Q}_{s}$,

$$
\begin{aligned}
& \sigma\left(x_{1}, f_{1}, \ldots, x_{n-1}, f_{n-1}\right)<\sigma\left(x_{1}, f_{1}, \ldots, x_{n}, f_{n}\right), \\
& \max \left\{\operatorname{range}\left(x_{n}\right) \cup \operatorname{range}\left(f_{n}\right)\right\} \leq m_{\sigma(\phi)}^{1 / 2} .
\end{aligned}
$$

Although $x_{i}, f_{i}$ are elements of $c_{00}$ their role in the space $X_{\text {ius }}$ we shall define is quite different. Namely $x_{i}$ will be elements of the space itself and $f_{i}$ elements of its dual $X_{\text {ius }}^{*}$. For similar reasons we shall denote the standard basis of $c_{00}$ either by $\left(e_{n}\right)_{n}$ or $\left(e_{n}^{*}\right)_{n}$.

Definition 2.1. A sequence $\phi=\left(x_{1}, f_{1}, \ldots, x_{2 k}, f_{2 k}\right) \in \mathbf{Q}_{s}$ is said to be a special sequence of length $2 k$ provided that

$$
x_{1}=\frac{1}{n_{2 j}} \sum_{l=1}^{n_{2 j}} e_{1, l}, \quad f_{1}=\frac{1}{m_{2 j}} \sum_{l=1}^{n_{2 j}} e_{1, l}^{*}
$$

for some $j \in \mathbb{N}$ such that $m_{2 j}^{1 / 2}>2 k$,

where $\left(e_{1, l}\right)_{l=1}^{n_{2 j}}$ is a subset of the standard basis of $c_{00}$ of cardinality $n_{2 j}$, and for every $1 \leq i \leq k$, setting $\phi_{i}=\left(x_{1}, f_{1}, \ldots, x_{i}, f_{i}\right)$, we have

$$
\left\|f_{2 i}\right\|_{\infty} \leq \frac{1}{m_{\sigma\left(\phi_{2 i-1}\right)}}, \quad\left|f_{2 i}\left(x_{2 i}\right)\right| \leq \frac{1}{m_{\sigma\left(\phi_{2 i-1}\right)}}
$$

and if $i<k$ then

$$
x_{2 i+1}=\frac{1}{n_{\sigma\left(\phi_{2 i}\right)}} \sum_{l=1}^{n_{\sigma\left(\phi_{2 i}\right)}} e_{2 i+1, l}, \quad f_{2 i+1}=\frac{1}{m_{\sigma\left(\phi_{2 i}\right)}} \sum_{l=1}^{n_{\sigma\left(\phi_{2 i}\right)}} e_{2 i+1, l}^{*},
$$

where for every $i \geq 1,\left(e_{2 i+1, l}\right)_{l=1}^{\left.n_{\sigma\left(\phi_{2 i}\right.}\right)}$ is a subset of the standard basis of $c_{00}$ of cardinality $n_{\sigma\left(\phi_{2 i}\right)}$.

The norming set of the space $X_{\text {ius }}$. The norming set $K$ will be the union $\bigcup_{n=0}^{\infty} K_{n}$, where the sequence $\left(K_{n}\right)_{n}$ is increasing and inductively defined as follows. We set

$$
K_{0}=\left\{ \pm e_{n}^{*}: n \in \mathbb{N}\right\}, \quad K_{0}^{j}=\emptyset \quad \text { for } j=1,2, \ldots
$$

Assume that $K_{n-1}=\bigcup_{j} K_{n-1}^{j}$ has been defined. Then for $j \in \mathbb{N}$ we set

$$
K_{n}^{2 j}=K_{n-1}^{2 j} \cup\left\{\frac{1}{m_{2 j}} \sum_{i=1}^{d} f_{i}: d \leq n_{2 j}, f_{1}<\ldots<f_{d}, f_{i} \in K_{n-1}\right\} .
$$


Moreover, for $j \in \mathbb{N}$ and every special sequence $\phi=\left(x_{1}, f_{1}, \ldots, x_{n_{2 j+1}}\right.$, $f_{n_{2 j+1}}$ ) of length $n_{2 j+1}$ (see Definition 2.1) such that $f_{2 i} \in K_{n-1}^{\sigma\left(\phi_{2 i-1}\right)}$ for $i=1, \ldots, n_{2 j+1} / 2\left(\right.$ where $\left.\phi_{2 i-1}=\left(x_{1}, f_{1}, \ldots, x_{2 i-1}, f_{2 i-1}\right)\right)$ we define

$$
\begin{array}{r}
K_{n, \phi}^{2 j+1}=\left\{\frac{ \pm 1}{m_{2 j+1}} E\left(\lambda_{f_{2}^{\prime}} f_{1}+f_{2}^{\prime}+\ldots+\lambda_{f_{n_{2 j+1}}^{\prime}} f_{n_{2 j+1}-1}+f_{n_{2 j+1}}^{\prime}\right):\right. \\
E \text { an interval of } \mathbb{N}, \operatorname{supp} f_{2 i}^{\prime}=\operatorname{supp} f_{2 i}, \\
f_{2 i}^{\prime} \in K_{n-1}^{\sigma\left(\phi_{2 i-1}\right)},\left|f_{2 i}^{\prime}\left(x_{2 i}\right)\right| \leq 1 / m_{\sigma\left(\phi_{2 i-1}\right)}, \\
\left.\lambda_{f_{2 i}^{\prime}}=f_{2 i}^{\prime}\left(m_{\sigma\left(\phi_{2 i-1}\right)} x_{2 i}\right) \text { if } f_{2 i}^{\prime}\left(x_{2 i}\right) \neq 0, \text { and } \pm 1 / n_{2 j+1}^{2} \text { otherwise }\right\} .
\end{array}
$$

Here, for $x=\sum_{i=1}^{\infty} a_{i} e_{i}$ and $E \subset \mathbb{N}$, we denote by $E x$ the vector $\sum_{i \in E} a_{i} e_{i}$. We define

$K_{n}^{2 j+1}=\bigcup\left\{K_{n, \phi}^{2 j+1}: \phi\right.$ is a special sequence of length $\left.n_{2 j+1}\right\} \cup K_{n-1}^{2 j+1}$, and finally we set

$$
K_{n}=\bigcup_{j} K_{n}^{j}
$$

This completes the inductive definition of $K_{n}$ and we set $K=\bigcup_{n} K_{n}$.

Observe that $K$ has the following properties:

(i) It is symmetric and $\|f\|_{\infty} \leq 1$ for each $f \in K$.

(ii) It is closed under interval projections (i.e. closed under restriction of its elements to intervals).

(iii) It is closed under the $\left(\mathcal{A}_{n_{2 j}}, 1 / m_{2 j}\right)$ operations (i.e. for $f_{1}<\ldots<f_{d}$ in $K$ with $d \leq n_{2 j}$ we have $\left.m_{2 j}^{-1} \sum_{l=1}^{d} f_{l} \in K\right)$.

(iv) If $f \in K$ then either $f= \pm e_{n}^{*}$ or $f \in K_{n}^{j}$ for some $n \geq 1, j \in \mathbb{N}$. In the latter case we define the weight of $f$ as $w(f)=m_{j}$. Note that $w(f)$ is not necessarily unique.

The space $X_{\text {ius }}$ is the completion of the space $\left(c_{00},\|\cdot\|_{K}\right)$, where

$$
\|x\|_{K}=\sup \{\langle f, x\rangle: f \in K\} \text {. }
$$

From the definition of $K$ it follows easily that $\left(e_{n}\right)_{n}$ is a bimonotone basis of $X_{\text {ius }}$. Also it is easy to see, by (iii), that the basis $\left(e_{n}\right)_{n}$ is boundedly complete. Indeed, for $x \in c_{00}$ and intervals $E_{1}<\ldots<E_{n_{2 j}}$ of $\mathbb{N}$ it follows from (iii) that

$$
\|x\| \geq \frac{1}{m_{2 j}} \sum_{i=1}^{n_{2 j}}\left\|E_{i} x\right\| .
$$

Also from the choice of the sequences $\left(n_{i}\right)_{i},\left(m_{i}\right)_{i}$ it follows that $n_{2 j} / m_{2 j}$ increases to infinity. These observations easily imply that the basis is boundedly complete. 
To prove that the space $X_{\text {ius }}$ is reflexive we need to show that the basis is shrinking. This requires some further work and we will present the argument later.

Lemma 2.2. Let $\phi=\left(x_{1}, f_{1}, \ldots, x_{n_{2 j+1}}, f_{n_{2 j+1}}\right)$ be a special sequence of length $n_{2 j+1}$ such that:

(a) $\left\{f_{i}: i=1, \ldots, n_{2 j+1}\right\} \subset K$ and $w\left(f_{i}\right)=m_{\sigma\left(\phi_{i-1}\right)}$ for $i \geq 2$.

(b) $\left\|w\left(f_{2 i}\right) x_{2 i}\right\| \leq 1$ for $1 \leq i \leq n_{2 j+1} / 2$

Then there exists $n \in \mathbb{N}$ such that $K_{n, \phi}^{2 j+1}$ is nonempty.

Notation. For every special sequence $\phi$ of length $n_{2 j+1}$ such that $K_{n, \phi}^{2 j+1}$ $\neq \emptyset$ for some $n$ we define $K_{\phi}=\bigcup_{n} K_{n, \phi}^{2 j+1}$.

REMARK 2.3. Let us point out that in the definition of the special sequences we have attempted to connect averages of the basis with block vectors that are quite freely chosen. This will be used to show that the quotient map from $X_{\text {ius }}$ to $X_{\text {ius }} /\left\langle e_{n}\right\rangle_{n \in M}$ is a strictly singular operator. Moreover we keep the dependence only between $f_{2 i-1}$ and the family $\{g \in K: w(g)=$ $\left.w\left(f_{2 i}\right), \operatorname{supp}(g)=\operatorname{supp}\left(f_{2 i}\right)\right\}$ to ensure that the space $X_{\text {ius }}$ is unconditionally saturated.

Definition 2.4 (The tree $\mathcal{T}_{f}$ of a functional $f \in K$ ). Let $f \in K$. We define a tree of $f$ (or tree corresponding to the analysis of $f$ ) to be every finite family $\mathcal{T}_{f}=\left(f_{\alpha}\right)_{\alpha \in \mathcal{A}}$ indexed by a finite tree $\mathcal{A}$ with a unique root $0 \in \mathcal{A}$ such that the following conditions are satisfied:

(1) $f_{0}=f$ and $f_{\alpha} \in K$ for each $\alpha \in \mathcal{A}$.

(2) If $\alpha \in \mathcal{A}$ is a terminal node then $f_{\alpha} \in K_{0}$.

(3) For every $\alpha \in \mathcal{A}$ which is not terminal, if we denote by $S_{\alpha}$ the set of immediate successors of $\alpha$, then exactly one of the following two conditions holds:

(a) $S_{\alpha}=\left\{\beta_{1}, \ldots, \beta_{d}\right\}$ with $f_{\beta_{1}}<\ldots<f_{\beta_{d}}$ and there exists $j \in \mathbb{N}$ such that $d \leq n_{2 j}$ and $f_{\alpha}=m_{2 j}^{-1} \sum_{i=1}^{d} f_{\beta_{i}}$.

(b) There exists a special sequence $\phi=\left(x_{1}, f_{1}, \ldots, x_{n_{2 j+1}}, f_{n_{2 j+1}}\right)$ of length $n_{2 j+1}$, an interval $E$ and $\varepsilon \in\{-1,1\}$ such that

$$
f_{\alpha}=\frac{\varepsilon}{m_{2 j+1}} \sum_{i=1}^{n_{2 j+1} / 2} E\left(\lambda_{f_{2 i}^{\prime}} f_{2 i-1}+f_{2 i}^{\prime}\right) \in K_{\phi}
$$

and $\left\{f_{\beta}: \beta \in S_{\alpha}\right\}=\left\{E f_{2 i-1}: E f_{2 i-1} \neq 0\right\} \cup\left\{E f_{2 i}^{\prime}: E f_{2 i}^{\prime} \neq 0\right\}$.

It follows from the inductive definition of $K$ that every $f \in K$ admits a tree, not necessarily unique. 
3. The space $X_{\text {ius }}$ is unconditionally saturated. We start by setting

$$
\widetilde{K}=\left\{ \pm e_{n}, \frac{1}{m_{2 j}} \sum_{i \in F} \pm e_{i}: \# F \leq n_{2 j}, j \in \mathbb{N}\right\} \cup\{0\} .
$$

Clearly $\widetilde{K}$ is a subset of the norming set $K$ and it is easily checked that $\widetilde{K}$ is a countable and compact (in the pointwise topology). It is well known that the space $C(\widetilde{K})$ is $c_{0}$-saturated. Observe also that $\|\cdot\|_{\widetilde{K}} \leq\|\cdot\|_{X_{\text {ius }}}$ and hence the identity operator

$$
I:\left(c_{00},\|\cdot\|_{X_{\text {ius }}}\right) \rightarrow\left(c_{00},\|\cdot\|_{\widetilde{K}}\right)
$$

is bounded. Since the basis $\left(e_{n}\right)_{n}$ of $X_{\text {ius }}$ is boundedly complete, the space $X_{\text {ius }}$ does not contain $c_{0}$, and therefore the operator $I$ is also strictly singular. These observations imply that every block subspace $Y$ of $X_{\text {ius }}$ contains a further block sequence $\left(y_{n}\right)$ such that $\left\|y_{n}\right\|_{X_{\text {ius }}}=1$ and $\left\|y_{n}\right\|_{\widetilde{K}} \stackrel{n}{\rightarrow} 0$. Our intention is to show the following:

Proposition 3.1. Let $\left(x_{l}\right)_{l}$ be a normalized block sequence in $X_{\text {ius }}$ such that $\left\|x_{l}\right\|_{\widetilde{K}} \rightarrow 0$. Then there exists a subsequence $\left(x_{l}\right)_{l \in M}$ of $\left(x_{l}\right)$ which is an unconditional basic sequence.

The proof of this proposition requires several steps and we sketch the main ideas. First we assume, upon passing to a subsequence, that $\left\|x_{l}\right\|_{\widetilde{K}}<\sigma_{l}$ with $\sum \sigma_{l}<1 / 8$, and we claim that $\left(x_{l}\right)_{l \in \mathbb{N}}$ is an unconditional basic sequence. Indeed, consider a norm one combination $\sum_{l=1}^{d} b_{l} x_{l}$ and let $\left(\varepsilon_{l}\right)_{l=1}^{d} \in$ $\{-1,1\}^{d}$. We shall show that $\left\|\sum_{l=1}^{d} \varepsilon_{l} b_{l} x_{l}\right\|>1 / 4$. Choose any $f \in K$ with $f\left(\sum_{l=1}^{d} b_{l} x_{l}\right)>3 / 4$; we are seeking a $g \in K$ such that $g\left(\sum_{l=1}^{d} \varepsilon_{l} b_{l} x_{l}\right) \geq 1 / 4$. To find such a $g$ a normal procedure is to consider a tree $\left(f_{\alpha}\right)_{\alpha \in \mathcal{A}}$ of the functional $f$ and then to produce inductively a functional $g$ with a tree $\left(g_{\alpha}\right)_{\alpha \in \mathcal{A}}$ such that

$$
\left|f\left(x_{l}\right)-g\left(\varepsilon_{l} x_{l}\right)\right|<2 \sigma_{l},
$$

which easily yields the desired result.

In most cases, producing $g_{\alpha}$ from $f_{\alpha}$ is straightforward. Essentially there exists only one case where we need to be careful: when $f_{\alpha} \in K_{\phi}$ for some special sequence $\phi$ (i.e. $f_{\alpha}= \pm m_{2 j+1}^{-1} E\left(\lambda_{f_{2}^{\prime}} f_{1}+f_{2}^{\prime}+\ldots+\lambda_{f_{n_{2 j+1}-1}^{\prime}} f_{n_{2 j+1}-1}+\right.$ $\left.f_{n_{2 j+1}}\right)$ ) and for some $i \leq n_{2 j+1} / 2$ and $l<d$ we have

$$
\begin{gathered}
\max \operatorname{supp} x_{l-1}<\min \operatorname{supp} f_{2 i-1} \leq \max \operatorname{supp} x_{l}, \\
\max \operatorname{supp} f_{2 i}^{\prime} \geq \min \operatorname{supp} x_{l+1} .
\end{gathered}
$$

In this case we produce $g_{\alpha}$ from $f_{\alpha}$ such that $g_{\alpha} \in K_{\phi}$. The form of $f_{\alpha}$ and hence $g_{\alpha}$ permits us to show that $\left|f_{\alpha}\left(x_{l}\right)-g_{\alpha}\left(\varepsilon_{l} x_{l}\right)\right|<2 \sigma_{l}$.

We now pass to the proof, starting with some notation and definitions. 
Notation. Let $f \in K$ and $\left(f_{\alpha}\right)_{\alpha \in \mathcal{A}}$ a tree of $f$. Then for every nonterminal node $\alpha \in \mathcal{A}$ we order the set $S_{\alpha}$ following the natural order of $\left\{\operatorname{supp} f_{\beta}\right\}_{\beta \in S_{\alpha}}$. For $\beta \in S_{\alpha}$ we denote by $\beta^{+}$the immediate successor of $\beta$ in the above order if such an object exists.

Definition 3.2. Let $f \in K$ and $\left(f_{\alpha}\right)_{\alpha \in \mathcal{A}}$ a tree of $f$. A couple of functionals $f_{\alpha}, f_{\alpha^{+}}$is said to be a dependent couple with respect to $f$ if there exists $\beta \in \mathcal{A}$ such that $\alpha, \alpha^{+} \in S_{\beta}$,

$$
f_{\beta}=\frac{\varepsilon}{m_{2 j+1}} E\left(\sum_{i=1}^{n_{2 j+1} / 2} \lambda_{f_{2 i}^{\beta}} f_{2 i-1}^{\beta}+f_{2 i}^{\beta}\right),
$$

and $f_{\alpha}=E f_{2 i-1}^{\beta}$ and $f_{\alpha^{+}}=E f_{2 i}^{\beta}$ for some $i \leq n_{2 j+1} / 2$.

Definition 3.3. Let $\left(x_{k}\right)_{k}$ be a normalized block sequence, $f \in K$ and $\mathcal{T}_{f}=\left(f_{\alpha}\right)_{\alpha \in \mathcal{A}}$ a tree of $f$. For $k \in \mathbb{N}$, a couple of functionals $f_{\alpha}, f_{\alpha^{+}}$is said to be a dependent couple with respect to $f$ and $x_{k}$ if $f_{\alpha}, f_{\alpha^{+}}$is a dependent couple w.r.t. $f$ and moreover

$$
\begin{gathered}
\max \operatorname{supp} x_{k-1}<\min \operatorname{supp} f_{\alpha} \leq \max \operatorname{supp} x_{k}, \\
\max \operatorname{supp} f_{\alpha^{+}} \geq \min \operatorname{supp} x_{k+1} .
\end{gathered}
$$

We also set

$$
\begin{aligned}
\mathcal{F}_{f, x_{k}}=\left\{\alpha \in \mathcal{A}: f_{\alpha}, f_{\alpha^{+}}\right. & \text {is a dependent couple w.r.t. } \left.f \text { and } x_{k}\right\}, \\
& \mathcal{F}_{f}=\bigcup_{k} \mathcal{F}_{f, x_{k}} .
\end{aligned}
$$

REMARK 3.4. Let $\left(x_{k}\right)$ be a block sequence in $X_{\text {ius }}, f \in K$ and $\left(f_{\alpha}\right)_{\alpha \in \mathcal{A}}$ a tree of $f$.

(1) It is easy to see that for every $k \in \mathbb{N}$ and every nonterminal node $\alpha \in \mathcal{A}$ the set $S_{\alpha} \cap \mathcal{F}_{f, x_{k}}$ has at most one element.

(2) As a consequence, for every $k$, any two elements $\alpha_{1}, \alpha_{2} \in \mathcal{F}_{f, x_{k}}$ with $\alpha_{1} \neq \alpha_{2}$ are incomparable and $\left|\alpha_{1}\right| \neq\left|\alpha_{2}\right|$, where we denote by $|\alpha|$ the order of $\alpha$ as a member of the finite tree $\mathcal{A}$.

(3) It is also easy to see that any $\alpha_{1}, \alpha_{2} \in \mathcal{F}_{f}$ with $\alpha_{1} \neq \alpha_{2}$ are incomparable and hence range $\left(f_{\alpha_{1}}\right) \cap \operatorname{range}\left(f_{\alpha_{2}}\right)=\emptyset$.

LEMmA 3.5. Let $\left(x_{k}\right)_{k}$ be a block sequence in $X_{\text {ius }}$ such that $\left\|x_{k}\right\|_{\widetilde{K}} \leq \sigma_{k}$, let $f \in K$ and $\left(f_{\alpha}\right)_{\alpha \in \mathcal{A}}$ a tree of $f$. Set $y_{k}=\left.x_{k}\right|_{\cup_{\alpha \in \mathcal{F}_{f}}} \operatorname{supp} f_{\alpha}$. Then

$$
\left|f\left(y_{k}\right)\right| \leq 2 \sigma_{k} \text {. }
$$

Proof. First observe that for each $q \in \mathbb{N}$ the sets range $\left(f_{\alpha}\right)$ with $|\alpha|=q$ are pairwise disjoint. Therefore from the preceding remark we deduce that for each $k$ and each $q$ the set

$$
\left\{\alpha \in \mathcal{F}_{f}:|\alpha|=q \text {, range }\left(f_{\alpha}\right) \cap \operatorname{range}\left(x_{k}\right) \neq \emptyset\right\}
$$


contains at most two elements (one of them belongs to $\mathcal{F}_{f, x_{k}}$ and the other to $\mathcal{F}_{f, x_{l}}$ for some $\left.l \leq k-1\right)$. Therefore

$$
\begin{aligned}
\left|f\left(y_{k}\right)\right| & \leq \sum_{\alpha \in \mathcal{F}_{f}}\left(\prod_{0 \preceq \gamma \prec \alpha} \frac{1}{w\left(f_{\gamma}\right)}\right)\left|f_{\alpha}\left(x_{k}\right)\right| \\
& =\sum_{i} \sum_{\alpha \in \mathcal{F}_{f},|\alpha|=i}\left(\prod_{0 \preceq \gamma \prec \alpha} \frac{1}{w\left(f_{\gamma}\right)}\right)\left|f_{\alpha}\left(x_{k}\right)\right| \leq 2 \sigma_{k} \sum_{i} \frac{1}{m_{1}^{i}} \leq 2 \sigma_{k} .
\end{aligned}
$$

The following lemma is the crucial step for the proof of the main result of this section.

LEMMA 3.6. Let $\left(x_{k}\right)_{k}$ be a block sequence in $X_{\text {ius }}, f \in K$ and $\left(f_{\alpha}\right)_{\alpha \in \mathcal{A}}$ a tree of $f$. For every $k \in \mathbb{N}$ set $y_{k}=\left.x_{k}\right|_{\bigcup_{\alpha \in \mathcal{F}_{f}}} \operatorname{supp} f_{\alpha}$. Then for every choice of signs $\left(\varepsilon_{k}\right)_{k}$ there exists a functional $g \in K$ with a tree $\left(g_{\alpha}\right)_{\alpha \in \mathcal{A}}$ such that:

(1) $f\left(x_{k}-y_{k}\right)=g\left(\varepsilon_{k}\left(x_{k}-y_{k}\right)\right)$,

(2) $\operatorname{supp} f_{\alpha}=\operatorname{supp} g_{\alpha}$ for every $\alpha \in \mathcal{A}$,

(3) $\mathcal{F}_{f, x_{k}}=\mathcal{F}_{g, x_{k}}$,

for every $k=1,2, \ldots$

Proof. For the given tree $\left(f_{\alpha}\right)_{\alpha \in \mathcal{A}}$ of $f$, we define $D=\left\{\beta \in \mathcal{A}: \operatorname{range}\left(f_{\beta}\right) \cap \operatorname{range}\left(x_{k}\right) \neq \emptyset\right.$ for at most one $k$ and if $\beta \in S_{\alpha}$ then range $\left(f_{\alpha}\right) \cap \operatorname{range}\left(x_{i}\right) \neq \emptyset$ for at least two $x_{i}$. Observe that for every branch $b$ of $\mathcal{A}, b \cap D$ is a singleton. Furthermore, for $\beta \in D$ and $\gamma \in \mathcal{A}$ with $\beta \prec \gamma$ we have $\gamma \notin \mathcal{F}_{f}$.

The definition of $\left(g_{\alpha}\right)_{\alpha \in \mathcal{A}}$ requires the following three steps.

STEP 1. First we define the set $\left\{g_{\beta}: \beta \in D\right\}$ as follows.

(a) If $\beta \in D$ and there exists $\alpha \in \mathcal{A}$ with $\alpha \preceq \beta$ and $f_{\alpha}, f_{\alpha^{+}}$is a dependent couple w.r.t. $f$ then we set $g_{\beta}=f_{\beta}$.

(b) If $\beta \in D$ does not fall under the previous case and there exists a (unique) $k$ such that $\operatorname{range}\left(f_{\beta}\right) \cap \operatorname{range}\left(x_{k}\right) \neq \emptyset$ then we set $g_{\beta}=\varepsilon_{k} f_{\beta}$.

(c) If $\beta \in D$ does not fall under case (a) and range $\left(f_{\beta}\right) \cap \operatorname{range}\left(x_{k}\right)=\emptyset$ for all $k$ then we set $g_{\beta}=\varepsilon_{k} f_{\beta}$, where

$$
k=\max \left\{l: \operatorname{range}\left(x_{l}\right)<\operatorname{range}\left(f_{\beta}\right)\right\} .
$$

(We have assumed that min range $\left(x_{1}\right) \leq \min$ range $(f)$.)

Let us comment on case (a) in the above definition. First we observe that the unique $\alpha \in \mathcal{A}$ witnessing that $\beta$ falls under case (a) satisfies the following: either $\alpha=\beta$ or $|\alpha|=|\beta|-1$. Moreover if this $\alpha$ does not belong to $\mathcal{F}_{f}$ then $\alpha=\beta$ and $\alpha^{+} \in D$. In this case, if we assume that there exists a (unique) $k$ such that range $\left(f_{\alpha}\right) \cap \operatorname{range}\left(x_{k}\right) \neq \emptyset$ then $g_{\alpha^{+}}$is defined by cases 
(b) or (c) and $g_{\alpha^{+}}=\varepsilon_{k} f_{\alpha^{+}}$for the specific $k$. All these are straightforward consequences of the corresponding definitions.

STEP 2. We set

$$
D^{+}=\{\gamma \in \mathcal{A}: \text { there exists } \beta \in D \text { with } \beta \prec \gamma\} \text {. }
$$

For $\gamma \in D^{+}$we set $g_{\gamma}=\varepsilon_{\beta} f_{\gamma}$, where $\beta$ is the unique element of $D$ with $\beta \prec \gamma$, and $\varepsilon_{\beta} \in\{-1,1\}$ is such that $g_{\beta}=\varepsilon_{\beta} f_{\beta}$.

Clearly for every $\beta \in D \cup D^{+},\left(g_{\gamma}\right)_{\beta \preceq \gamma}$ is a tree of the functional $g_{\beta}$. Furthermore for $\alpha \in D \cup D^{+}$the following properties hold:

(1) $\operatorname{supp} f_{\alpha}=\operatorname{supp} g_{\alpha}$.

(2) $w\left(f_{\alpha}\right)=w\left(g_{\alpha}\right)$.

Step 3. We set

$$
D^{-}=\{\alpha \in \mathcal{A}: \text { there exists } \beta \in D \text { with } \alpha \prec \beta\} .
$$

Observe that $\mathcal{A}=D \cup D^{+} \cup D^{-}$. Using backward induction, for all $\alpha \in D^{-}$ we shall define $g_{\alpha}$ such that the above (1) and (2) hold, together with the following two properties:

(3) For $\alpha \in D^{-}$we have $f_{\alpha}\left(x_{k}-y_{k}\right)=g_{\alpha}\left(\varepsilon_{k}\left(x_{k}-y_{k}\right)\right)$ for all $k$.

(4) For $\alpha \in D^{-}$and each $k$ we have $\mathcal{F}_{f_{\alpha}, x_{k}}=\mathcal{F}_{g_{\alpha}, x_{k}}$.

Observe that $f_{\alpha} \notin K_{0}$ for every $\alpha \in D^{-}$and furthermore $\mathcal{F}_{f_{\beta}}=\emptyset$ for every $\beta \in D$.

We now pass to the inductive construction of $g_{\alpha}, \alpha \in D^{-}$, and to establishing properties (1)-(4). Assume that $\alpha \in D^{-}$and for every $\beta \in S_{\alpha}$ either $\beta \in D$ or $g_{\beta}$ has been defined and properties (1)-(4) have been established. We consider the following three cases.

CAse 1. $w\left(f_{\alpha}\right)=m_{2 j}$ and $\alpha \in \mathcal{F}_{f}$.

That means that $f_{\alpha}=m_{2 j}^{-1} \sum_{\beta \in S_{\alpha}} f_{\beta}$ and each $f_{\beta}$ is $e_{l}^{*}$ for some $l \in \mathbb{N}$. Then $S_{\alpha} \subset D$ and from Step 1(a) we conclude that $g_{\beta}=f_{\beta}$ for all $\beta \in S_{\alpha}$. We set

$$
g_{\alpha}=\frac{1}{m_{2 j}} \sum_{\beta \in S_{\alpha}} g_{\beta}=f_{\alpha}
$$

Furthermore for each $k$ we have $\operatorname{supp} g_{\alpha} \cap \operatorname{supp} x_{k} \subset \operatorname{supp} y_{k}$. Hence

$$
g_{\alpha}\left(\varepsilon_{k}\left(x_{k}-y_{k}\right)\right)=f_{\alpha}\left(x_{k}-y_{k}\right)=0
$$

and also $\mathcal{F}_{g_{\alpha}}=\mathcal{F}_{f_{\alpha}}=\emptyset$. Thus properties (3) and (4) hold while (1) and (2) are obvious.

Before passing to the next case notice that there is no $\alpha \in D^{-}$such that $f_{\alpha}, f_{\alpha^{+}}$is a dependent couple w.r.t. $f$ and $\alpha \notin \mathcal{F}_{f}$. (See the comments after Step 1.)

CASE 2. $w\left(f_{\alpha}\right)=m_{2 j}$ and $\alpha \notin \mathcal{F}_{f}$. 
From the previous observation we see that $\alpha \neq \beta$ for each $\beta \in \mathcal{A}$ with $f_{\beta}, f_{\beta^{+}}$a dependent couple w.r.t. $f$, and we set

$$
g_{\alpha}=\frac{1}{m_{2 j}} \sum_{\beta \in S_{\alpha}} g_{\beta} .
$$

Our inductive assumptions yield properties (1) and (2). To establish (3) let $k \in \mathbb{N}$ and $\beta \in D \cap S_{\alpha}$ be such that $\operatorname{range}\left(x_{k}\right) \cap \operatorname{range}\left(f_{\beta}\right) \neq \emptyset$. Then $g_{\beta}=\varepsilon_{k} f_{\beta}$ and hence

$$
g_{\beta}\left(\varepsilon_{k}\left(x_{k}-y_{k}\right)\right)=\varepsilon_{k} g_{\beta}\left(x_{k}-y_{k}\right)=f_{\beta}\left(x_{k}-y_{k}\right) .
$$

If $\beta \in D^{-} \cap S_{\alpha}$, by the inductive assumption for each $k$ we have

$$
g_{\beta}\left(\varepsilon_{k}\left(x_{k}-y_{k}\right)\right)=f_{\beta}\left(x_{k}-y_{k}\right) .
$$

Therefore

$$
g_{\alpha}\left(\varepsilon_{k}\left(x_{k}-y_{k}\right)\right)=f_{\alpha}\left(x_{k}-y_{k}\right) .
$$

Finally, for each $k$,

$$
\mathcal{F}_{f_{\alpha}, x_{k}}=\bigcup_{\beta \in S_{\alpha}} \mathcal{F}_{f_{\beta}, x_{k}}=\bigcup_{\beta \in S_{\alpha} \cap D^{-}} \mathcal{F}_{f_{\beta}, x_{k}}=\bigcup_{\beta \in S_{\alpha} \cap D^{-}} \mathcal{F}_{g_{\beta}, x_{k}}=\mathcal{F}_{g_{\alpha}, x_{k}},
$$

which establishes property (4).

CASE 3. $f_{\alpha}=\frac{\varepsilon}{m_{2 j+1}} E\left(\lambda_{f_{2}^{\alpha}} f_{1}^{\alpha}+f_{2}^{\alpha}+\ldots+\lambda_{f_{n_{2 j+1}}^{\alpha}} f_{n_{2 j+1}-1}^{\alpha}+f_{n_{2 j+1}}^{\alpha}\right) \in K_{\phi}$ where $\left\{f_{\beta}: \beta \in S_{\alpha}\right\}=\left\{E f_{i}^{\alpha}: E f_{i}^{\alpha} \neq 0,1 \leq i \leq n_{2 j+1}\right\}, \varepsilon \in\{-1,1\}, E$ is an interval and $\phi$ is a special sequence of length $n_{2 j+1}$.

Let $\phi=\left(z_{1}, f_{1}, \ldots, z_{n_{2 j+1}}, f_{n_{2 j+1}}\right)$. We can assume that $E=\mathbb{N}$ and $\varepsilon=1$. Observe that the definition of $\left\{g_{\beta}: \beta \in D\right\}$ and the inductive assumptions imply that for $i \leq n_{2 j+1} / 2$ :

(i) $f_{2 i-1}=f_{2 i-1}^{\alpha}=g_{2 i-1}^{\alpha}$.

(ii) $w\left(f_{2 i}\right)=w\left(f_{2 i}^{\alpha}\right)=w\left(g_{2 i}^{\alpha}\right)$.

(iii) $\operatorname{supp} f_{2 i}=\operatorname{supp} f_{2 i}^{\alpha}=\operatorname{supp} g_{2 i}^{\alpha}$.

We define

$$
g_{\alpha}=\frac{1}{m_{2 j+1}}\left(\lambda_{g_{2}^{\alpha}} f_{1}+g_{2}^{\alpha}+\lambda_{g_{4}^{\alpha}} f_{3}+g_{4}^{\alpha}+\ldots+\lambda_{g_{n_{2 j+1}}^{\alpha}} f_{n_{2 j+1}-1}+g_{n_{2 j+1}}^{\alpha}\right),
$$

where $\left\{g_{\beta}: \beta \in S_{\alpha}\right\}=\left\{g_{i}^{\alpha}: 1 \leq i \leq n_{2 j+1}\right\}$, while $\lambda_{g_{2 i}^{\alpha}}$ are defined as follows:

(5) If $g_{2 i}^{\alpha}\left(z_{2 i}\right) \neq 0$ then $\lambda_{g_{2 i}^{\alpha}}=g_{2 i}^{\alpha}\left(m_{\sigma\left(\phi_{2 i-1}\right)} z_{2 i}\right)$.

(6) If $g_{2 i}^{\alpha}\left(z_{2 i}\right)=0$ and $f_{2 i-1}^{\alpha}=f_{\beta}$, there are two cases:

(a) If $\beta \in \mathcal{F}_{f}$, or $\beta \notin \mathcal{F}_{f}$ and range $\left(f_{\beta}\right) \cap \operatorname{range}\left(x_{k}\right)=\emptyset$ for all $k$, we set $\lambda_{g_{2 i}^{\alpha}}^{\alpha}=1 / n_{2 j+1}^{2}$.

(b) If $\beta \notin \mathcal{F}_{f}$ and there exists a (unique) $k$ such that range $\left(f_{\beta}\right) \cap$ range $\left(x_{k}\right) \neq \emptyset$ then we set $\lambda_{g_{2 i}^{\alpha}}=\varepsilon_{k} \lambda_{f_{2 i}^{\alpha}}$. 
Observe that in the case (6)(b), as follows from the comments after Step 1, $g_{\beta^{+}}=\varepsilon_{k} f_{\beta^{+}}$, hence $f_{\beta^{+}}\left(z_{2 i}\right)=0$ if and only if $g_{\beta^{+}}\left(z_{2 i}\right)=0$.

From the above definition of $\lambda_{g_{2 i}^{\alpha}}, 1 \leq i \leq n_{2 j+1} / 2$, and (i)-(iii), we find that the functional $g_{\alpha}$ belongs to $K_{\phi} \subset K$.

Properties (1) and (2) are obvious for $g_{\alpha}$; we now check the rest. First we establish property (4).

Let $k$ be given. From Remark 3.4(1) it follows that there exists at most one dependent couple $f_{2 i-1}^{\alpha}, f_{2 i}^{\alpha}$ w.r.t. $f$ and $x_{k}$. Moreover, if such a couple exists then $\mathcal{F}_{f_{2}^{\prime}}^{\alpha}, x_{k}=\emptyset$ for every $i^{\prime} \neq i$. Therefore in this case

$$
\mathcal{F}_{f_{\alpha}, x_{k}}=\mathcal{F}_{f_{2 i}^{\alpha}, x_{k}} \cup\{\beta\},
$$

where $f_{2 i-1}^{\alpha}=f_{\beta}$. If no such dependent couple exists, it follows that $\mathcal{F}_{f_{2 i}, x_{k}}$ $\neq \emptyset$ for at most one $i$. This is a consequence of the definitions and the fact that the functionals $\left(f_{i}^{\alpha}\right)_{i}$ are successive. If such an $i$ exists then

$$
\mathcal{F}_{f_{\alpha}, x_{k}}=\mathcal{F}_{f_{2 i}^{\alpha}, x_{k}} .
$$

The last alternative is that $\mathcal{F}_{f_{\alpha}, x_{k}}=\emptyset$. This description of $\mathcal{F}_{f_{\alpha}, x_{k}}$ and the inductive assumptions easily yield property (4). Namely, either

$$
\mathcal{F}_{g_{\alpha}, x_{k}}=\mathcal{F}_{g_{2 i}^{\alpha}, x_{k}} \cup\{\beta\}
$$

if (3.5) holds, or $\mathcal{F}_{g_{\alpha}, x_{k}}=\mathcal{F}_{g_{2 i}^{\alpha}, x_{k}}$ if (3.6) holds, or $\mathcal{F}_{g_{\alpha}, x_{k}}=\emptyset$.

Finally, we check property (3). Fix $k$ and $i \leq n_{2 j+1} / 2$. If $g_{2 i}^{\alpha}=g_{\beta}$ and $\beta \in D^{-}$the inductive assumption provides

$$
g_{2 i}^{\alpha}\left(\varepsilon_{k}\left(x_{k}-y_{k}\right)\right)=f_{2 i}^{\alpha}\left(x_{k}-y_{k}\right) .
$$

If $\beta \in D$ and range $\left(f_{2 i}^{\alpha}\right) \cap \operatorname{range}\left(x_{k}\right) \neq \emptyset$ then $g_{2 i}^{\alpha}=\varepsilon_{k} f_{2 i}^{\alpha}$, which yields (3.7). Also if range $\left(f_{2 i}^{\alpha}\right) \cap \operatorname{range}\left(x_{k}\right)=\emptyset$ equality (3.7) trivially holds.

In the case $g_{2 i-1}^{\alpha}=g_{\beta}, \beta \in S_{\alpha}$, we distinguish two subcases. First assume that $\beta \in \mathcal{F}_{f}$. Then $\operatorname{supp} g_{2 i-1}^{\alpha}=\operatorname{supp} f_{2 i-1}^{\alpha}$ and $\operatorname{supp} f_{2 i-1}^{\alpha} \cap \operatorname{supp}\left(x_{k}-y_{k}\right)$ $=\emptyset$, therefore

$$
g_{2 i-1}^{\alpha}\left(\varepsilon_{k}\left(x_{k}-y_{k}\right)\right)=0=f_{2 i-1}^{\alpha}\left(x_{k}-y_{k}\right) .
$$

The second subcase is $\beta \notin \mathcal{F}_{f}$. As explained in the comments after Step 1, that means that either range $\left(f_{\beta}\right) \cap \operatorname{range}\left(x_{k}\right)=\emptyset$, hence everything trivially holds, or $\beta, \beta^{+} \in D, g_{\beta^{+}}=\varepsilon_{k} f_{\beta^{+}}$and $\lambda_{g_{2 i}^{\alpha}}=\varepsilon_{k} \lambda_{f_{2 i}^{\alpha}}$. From these observations we conclude that

$$
\lambda_{g_{2 i}^{\alpha}} g_{2 i-1}^{\alpha}\left(\varepsilon_{k}\left(x_{k}-y_{k}\right)\right)=\lambda_{f_{2 i}^{\alpha}} f_{2 i-1}^{\alpha}\left(x_{k}-y_{k}\right) .
$$

All these yield the desired equality, namely

$$
g_{\alpha}\left(\varepsilon_{k}\left(x_{k}-y_{k}\right)\right)=f_{\alpha}\left(x_{k}-y_{k}\right) .
$$

The inductive construction and the entire proof of the lemma are complete.

Proof of Proposition 3.1. Let $\left(\sigma_{l}\right)_{l}$ be a decreasing sequence of positive numbers such that $\sum_{l} \sigma_{l} \leq 1 / 8$. For each $l \in \mathbb{N}$ we select $k_{l}$ such that 
$\left\|x_{k_{l}}\right\|_{\widetilde{K}}<\sigma_{l}$. For simplicity we assume that the entire sequence $\left(x_{l}\right)$ satisfies the above condition. Let $\sum_{l=1}^{d} b_{l} x_{l}$ be a finite linear combination which maximizes the norm of all vectors of the form $\sum_{l=1}^{d} c_{l} x_{l}$ with $\left|c_{l}\right|=\left|b_{l}\right|$. Assume furthermore that $\left\|\sum_{l=1}^{d} b_{l} x_{l}\right\|=1$ and let $f \in K$ with $f\left(\sum_{l=1}^{d} b_{l} x_{l}\right) \geq 3 / 4$. Choose $\left\{\varepsilon_{l}\right\}_{l=1}^{d} \in\{-1,1\}^{d}$ and consider the vector $\sum_{l=1}^{d} \varepsilon_{l} b_{l} x_{l}$. Lemma 3.6 shows that there exists $g \in K$ and, for each $l=1, \ldots, d$, a vector $y_{l}$ such that

$$
g\left(\sum_{l=1}^{d} \varepsilon_{l} b_{l}\left(x_{l}-y_{l}\right)\right)=f\left(\sum_{l=1}^{d} b_{l}\left(x_{l}-y_{l}\right)\right) .
$$

Also Lemmas 3.5 and 3.6(2), (3) yield

$$
\left|g\left(y_{l}\right)\right| \leq 2 \sigma_{l}, \quad\left|f\left(y_{l}\right)\right| \leq 2 \sigma_{l} \quad \text { for all } l=1, \ldots, d .
$$

Hence

$$
\begin{aligned}
\left\|\sum_{l=1}^{d} \varepsilon_{l} b_{l} x_{l}\right\| & \geq\left|g\left(\sum_{l=1}^{d} \varepsilon_{l} b_{l} x_{l}\right)\right| \geq\left|g\left(\sum_{l=1}^{d} \varepsilon_{l} b_{l}\left(x_{l}-y_{l}\right)\right)\right|-\sum_{\ell=1}^{d}\left|g\left(y_{l}\right)\right| \\
& \geq\left|f\left(\sum_{l=1}^{d} b_{l} x_{l}\right)\right|-\sum_{l=1}^{d}\left|g\left(y_{l}\right)\right|-\sum_{l=1}^{d}\left|f\left(y_{l}\right)\right| \\
& \geq 3 / 4-2 / 4=1 / 4 .
\end{aligned}
$$

This completes the proof of the proposition.

4. The space $X_{\text {ius }}$ is indecomposable. In the last section we shall show that the space $X_{\text {ius }}$ is indecomposable. This will be a consequence of a stronger result concerning the structure of the space $\mathcal{B}\left(X_{\text {ius }}\right)$ of bounded linear operators acting on $X_{\text {ius }}$. The proof adapts techniques related to H.I. spaces as presented in [AT1]. Thus we will first consider the auxiliary space $X_{\mathrm{u}}$ and we will estimate the norm of certain averages of its basis. Next we will use the basic inequality to reduce upper estimation of certain averages to the previous results. Finally, we shall compute the norms of linear combinations related to special sequences.

The auxiliary spaces $X_{\mathrm{u}}, X_{\mathrm{u}, k}$. We begin with the definition of the space $X_{\mathrm{u}}$ which will be used to provide us with upper estimates for certain averages in the space $X_{\text {ius }}$.

The space $X_{\mathrm{u}}$ is the mixed Tsirelson space $T\left[\left(\mathcal{A}_{4 n_{j}}, 1 / m_{j}\right)_{j=1}^{\infty}\right]$. The norming set $W$ of $X_{\mathrm{u}}$ is defined in a similar manner to the set $K$.

We set $W_{0}^{j}=\left\{ \pm e_{n}^{*}: n \in \mathbb{N}\right\} \cup\{0\}$ for $j \in \mathbb{N}$, and $W_{0}=\bigcup_{j} W_{0}^{j}$. In the general inductive step we define 


$$
W_{n}^{j}=W_{n-1}^{j} \cup\left\{\frac{1}{m_{j}} \sum_{i=1}^{d} f_{i}: d \leq 4 n_{j}, f_{1}<\ldots<f_{d} \in W_{n-1}\right\}
$$

and $W_{n}=\bigcup_{j} W_{n}^{j}$. Finally, let $W=\bigcup_{n} W_{n}$. The space $X_{\mathrm{u}}$ is the completion of $\left(c_{00},\|\cdot\|_{W}\right)$, where

$$
\|x\|_{W}=\sup \{\langle f, x\rangle: f \in W\} .
$$

It is clear that the norming set $K$ of the space $X_{\text {ius }}$ is a subset of the convex hull of $W$. Hence $\|x\|_{K} \leq\|x\|_{W}$ for every $x \in c_{00}$.

We also need the spaces $X_{\mathrm{u}, k}=T\left[\left(\mathcal{A}_{4 n_{j}}, 1 / m_{j}\right)_{j=1}^{k}\right]$. The norm of such a space is denoted by $\|\cdot\|_{\mathrm{u}, k}$ and it is defined in a similar manner to the norm of $X_{\mathrm{u}}$. Namely we define $W_{n}^{j}, n \in \mathbb{N}, 1 \leq j \leq k$, as above and $W_{n}^{(k)}=\bigcup_{j=1}^{k} W_{n}^{j}$. The norming set is $W^{(k)}=\bigcup_{n=0}^{\infty} W_{n}^{(k)}$. Spaces of this form have been studied in [BD] and it has been shown that such a space is isomorphic either to some $\ell_{p}, 1<p<\infty$, or to $c_{0}$.

Before stating the next lemma we introduce some notations. For each $k \in \mathbb{N}$ we set

$$
q_{k}=\frac{1}{\log _{4 n_{k}} m_{k}}, \quad p_{k}=\frac{1}{1-\log _{4 n_{k}} m_{k}} .
$$

Lemma 4.1. For the sequences $\left(m_{j}\right)_{j},\left(n_{j}\right)_{j}$ used in the definition of $X_{\mathrm{ius}}$ and $X_{\mathrm{u}}, X_{\mathrm{u}, k}$ the following hold:

(1) The sequence $\left(q_{j}\right)_{j}$ strictly increases to infinity.

(2) For $x=\sum a_{l} e_{l} \in c_{00},\|x\|_{\mathrm{u}, k} \leq\|x\|_{p_{k}}$.

(3) $\left\|\frac{1}{n_{k+1}} \sum_{i=1}^{n_{k+1}} e_{i}\right\|_{p_{k}} \leq \frac{1}{m_{k+1}^{3}}$.

Proof. (1) Using the facts that $m_{j+1}=m_{j}^{5}, n_{j+1}=\left(4 n_{j}\right)^{s_{j}}$ and $s_{j}$ increases to infinity, we find that

$$
q_{j+1}=\frac{1}{\log _{4 n_{j+1}} m_{j+1}}=\frac{1}{\log _{4\left(4 n_{j}\right)^{s_{j}} m_{j}^{5}}}>\frac{1}{\frac{5}{s_{j}} \log _{4 n_{j}} m_{j}}=\frac{s_{j}}{5} q_{j},
$$

hence $\left(q_{j}\right)_{j}$ strictly increases to infinity.

(2) We inductively show that for $f \in W_{n}^{(k)}$,

$$
\left|f\left(\sum a_{l} e_{l}\right)\right| \leq\left\|\sum a_{l} e_{l}\right\|_{p_{k}} .
$$

For $n=0$ this is trivial. The general inductive step goes as follows: for $f \in W_{n+1}^{(k)}$,

$$
f\left(\sum a_{l} e_{l}\right)=\frac{1}{m_{j}} \sum_{i=1}^{d} f_{i}\left(\sum a_{l} e_{l}\right)
$$


where $f_{1}<\ldots<f_{d}$ and $d \leq 4 n_{j}$ for some $j \leq k$. We set $E_{i}=\operatorname{range}\left(f_{i}\right)$ and from our inductive assumption and the Hölder inequality we obtain

$$
\left|f\left(\sum a_{l} e_{l}\right)\right| \leq \frac{1}{m_{j}} \sum_{i=1}^{d}\left\|\sum_{\ell \in E_{i}} a_{l} e_{l}\right\|_{p_{k}} \leq \frac{d^{1 / q_{j}}}{m_{j}}\left(\sum_{i=1}^{d}\left\|\sum_{l \in E_{i}} a_{l} e_{l}\right\|_{p_{k}}^{p_{j}}\right)^{1 / p_{j}} .
$$

Making use of $p_{k} \leq p_{j}$ and $m_{j}=\left(4 n_{j}\right)^{1 / q_{j}}$ we obtain inequality (2).

(3) We have

$$
\left\|\frac{1}{n_{k+1}} \sum_{i=1}^{n_{k+1}} e_{i}\right\|_{p_{k}} \leq \frac{1}{n_{k+1}^{1 / q_{k}}}=\frac{1}{\left(4 n_{k}\right)^{s_{k} / q_{k}}}=\frac{1}{m_{k}^{s_{k}}} \leq \frac{1}{m_{k+1}^{3}} .
$$

(Recall that $2^{s_{k}} \geq m_{k+1}^{3}$.)

The tree $\mathcal{T}_{f}$ of $f \in W$ is defined in a similar manner to that for $f \in K$.

Lemma 4.2. Let $f \in W$ and $j \in \mathbb{N}$. Then

$$
\left|f\left(\frac{1}{n_{j}} \sum_{i=1}^{n_{j}} e_{k_{i}}\right)\right| \leq \begin{cases}\frac{2}{w(f) \cdot m_{j}} & \text { if } w(f)<m_{j}, \\ \frac{1}{w(f)} & \text { if } w(f) \geq m_{j} .\end{cases}
$$

If moreover there exists a tree $\left(f_{\alpha}\right)_{\alpha \in \mathcal{A}}$ of $f$ such that $w\left(f_{\alpha}\right) \neq m_{j}$ for every $\alpha \in \mathcal{A}$, then

$$
\left|f\left(\frac{1}{n_{j}} \sum_{i=1}^{n_{j}} e_{k_{i}}\right)\right| \leq \frac{2}{m_{j}^{3}} .
$$

In particular the above upper estimates hold for every $f \in K$.

Proof. If $w(f) \geq m_{j}$ the estimate is an immediate consequence of the fact that $\|f\|_{\infty} \leq 1 / w(f)$. Assume $w(f)<m_{j}$ and let $\left(f_{\alpha}\right)_{\alpha \in \mathcal{A}}$ be a tree of $f$. We set

$$
B=\left\{i \text { : there exists } \alpha \in \mathcal{A} \text { with } k_{i} \in \operatorname{supp} f_{\alpha} \text { and } w\left(f_{\alpha}\right) \geq m_{j}\right\} .
$$

Then

$$
\left|f\left(\frac{1}{n_{j}} \sum_{i \in B} e_{k_{i}}\right)\right| \leq \frac{1}{w(f) m_{j}} .
$$

To estimate $\left|f\left(n_{j}^{-1} \sum_{i \in B^{\mathrm{c}}} e_{k_{i}}\right)\right|$, we observe that $\left.f\right|_{\left\{k_{i}: i \in B^{\mathrm{c}}\right\}} \in W^{(j-1)}$ (the norming set of $\left.X_{\mathrm{u}, j-1}\right)$ and hence Lemma 4.1 yields

$$
\left|f\left(\frac{1}{n_{j}} \sum_{i \in B^{\mathrm{c}}} e_{k_{i}}\right)\right| \leq \frac{1}{m_{j}^{3}} .
$$

Combining (4.3) and (4.4) we obtain (4.1).

To prove (4.2) we define

$$
B=\left\{i \text { : there exists } \alpha \in \mathcal{A} \text { with } k_{i} \in \operatorname{supp} f_{\alpha} \text { and } w\left(f_{\alpha}\right) \geq m_{j+1}\right\}
$$


and we conclude that

$$
\left|f\left(\frac{1}{n_{j}} \sum_{i \in B} e_{k_{i}}\right)\right| \leq \frac{1}{m_{j+1}}<\frac{1}{m_{j}^{3}} .
$$

Furthermore from our assumption $w\left(f_{\alpha}\right) \neq m_{j}$ for every $\alpha \in \mathcal{A}$ we conclude that $\left.f\right|_{\left\{k_{i}: i \in B^{\mathrm{c}}\right\}} \in W^{(j-1)}$. This yields that (4.4) remains valid, and combining (4.4) and (4.5) we obtain (4.2).

The basic inequality and its consequences. Next we state and prove a basic inequality which is an adaptation of the corresponding result from [AT1]. Actually the proof of the present statement is easier than the original one, due mainly to the low complexity of the family $\mathcal{A}_{n}$ ([AT1] studies spaces defined with the use of the Schreier families $\left.\left(\mathcal{S}_{\xi}\right)_{\xi<\omega_{1}}\right)$ and also since the definition of the norming set $K$ does not involve convex combinations. This result is important since it includes most of the necessary computations (unconditional or conditional).

Recall that $K$ and $W$ denote the norming sets of $X_{\text {ius }}$ and $X_{\mathrm{u}}$ respectively.

Proposition 4.3 (Basic inequality). Let $\left(x_{k}\right)$ be a block sequence in $X_{\text {ius }}$, $\left(j_{k}\right)$ a strictly increasing sequence of positive integers, $\left(b_{k}\right) \in c_{00}, C \geq 1$ and $\varepsilon>0$ such that:

(a) $\left\|x_{k}\right\| \leq C$ for every $k$.

(b) \# $\left(\operatorname{supp} x_{k}\right) / m_{j_{k+1}} \leq \varepsilon$ for every $k$.

(c) $\left|f\left(x_{k}\right)\right| \leq C / w(f)$ for every $k$ and all $f \in K$ with $w(f)<m_{j_{k}}$.

Then for every $f \in K$ there exist $g_{1}$ such that $g_{1}=h_{1}$ or $g_{1}=e_{t}^{*}+h_{1}$, where $t \notin \operatorname{supp} h_{1}, h_{1} \in W, w\left(h_{1}\right)=w(f)$, and $g_{2} \in c_{00}$ with $\left\|g_{2}\right\|_{\infty} \leq \varepsilon$ such that

$$
\left|f\left(\sum b_{k} x_{k}\right)\right| \leq C\left(g_{1}+g_{2}\right)\left(\sum\left|b_{k}\right| e_{k}\right) \text {, }
$$

and $\operatorname{supp} g_{1}$, supp $g_{2}$ are contained in $\left\{k\right.$ : supp $\left.f \cap \operatorname{range}\left(x_{k}\right) \neq \emptyset\right\}$.

(d) If additionally, for some $j_{0} \in \mathbb{N}$, we have

$$
\left|f\left(\sum_{k \in E} b_{k} x_{k}\right)\right| \leq C\left(\max _{k \in E}\left|b_{k}\right|+\varepsilon \sum_{k \in E}\left|b_{k}\right|\right)
$$

for every interval $E$ of positive integers and every $f \in K$ with $w(f)=m_{j_{0}}$, then $h_{1}$ may be selected to have a tree $\left(h_{\alpha}\right)_{\alpha \in \mathcal{A}_{1}}$ such that $w\left(h_{\alpha}\right) \neq m_{j_{0}}$ for every $\alpha \in \mathcal{A}_{1}$.

Our intention is to apply the above inequality in order to obtain upper estimates for $\ell_{1}$ averages of rapidly increasing sequences. Observe that the above proposition reduces this problem to estimating the functionals $g_{1}, g_{2}$ on a corresponding average of the basis in $X_{\mathrm{u}}$. 
The proof in the general case, assuming only (a)-(c), and in the special case, where additionally (d) is assumed, is the same. We will give the proof only in the special case. The proof in the general case is obtained by omitting any reference to the question whether a functional has weight $m_{j_{0}}$ or not. For the rest of the proof we assume that there exists $j_{0} \in \mathbb{N}$ such that condition (d) in the statement of the proposition is satisfied.

Proof of Proposition 4.3. Let $f \in K$ and let $\mathcal{T}_{f}=\left(f_{\alpha}\right)_{\alpha \in \mathcal{A}}$ be a tree of $f$. For every $k$ such that $\operatorname{supp} f \cap \operatorname{range}\left(x_{k}\right) \neq \emptyset$ we define

$A_{k}=\left\{\alpha \in \mathcal{A}:(\mathrm{i}) \operatorname{supp} f_{\alpha} \cap \operatorname{range}\left(x_{k}\right)=\operatorname{supp} f \cap \operatorname{range}\left(x_{k}\right)\right.$,

(ii) $w\left(f_{\gamma}\right) \neq m_{j_{0}}$ for all $\gamma \prec \alpha$,

(iii) there is no $\beta \in S_{\alpha}$ such that

$$
\left.\operatorname{supp} f_{\alpha} \cap \operatorname{range}\left(x_{k}\right)=\operatorname{supp} f_{\beta} \cap \operatorname{range}\left(x_{k}\right) \text { if } w\left(f_{\alpha}\right) \neq m_{j_{0}}\right\} \text {. }
$$

From the definition, it follows easily that for every $k$ such that supp $f \cap$ range $\left(x_{k}\right) \neq \emptyset, A_{k}$ is a singleton.

We recursively define sets $\left(D_{\alpha}\right)_{\alpha \in \mathcal{A}}$ as follows.

For every terminal node $\alpha$ of the tree we set $D_{\alpha}=\left\{k: \alpha \in A_{k}\right\}$. For every nonterminal node $\alpha$ we define

$$
D_{\alpha}=\left\{k: \alpha \in A_{k}\right\} \cup \bigcup_{\beta \in S_{\alpha}} D_{\beta} .
$$

The following are easy consequences of the definition:

(i) If $\beta \prec \alpha, D_{\alpha} \subset D_{\beta}$.

(ii) If $w\left(f_{\alpha}\right)=m_{j_{0}}$, then $D_{\beta}=\emptyset$ for all $\beta \succ \alpha$.

(iii) If $w\left(f_{\alpha}\right) \neq m_{j_{0}}$, then $\left\{\{k\}: k \in D_{\alpha} \backslash \bigcup_{\beta \in S_{\alpha}} D_{\beta}\right\} \cup\left\{D_{\beta}: \beta \in S_{\alpha}\right\}$ is a family of successive subsets of $\mathbb{N}$.

(iv) If $w\left(f_{\alpha}\right) \neq m_{j_{0}}$, then for every $k \in D_{\alpha} \backslash \bigcup_{\beta \in S_{\alpha}} D_{\beta}$ there exists $\beta \in S_{\alpha}$ such that $\min \operatorname{supp} x_{k}<\min \operatorname{supp} f_{\beta} \leq \max \operatorname{supp} x_{k}$ and for $k^{\prime} \in$ $D_{\alpha} \backslash \bigcup_{\beta \in S_{\alpha}} D_{\beta}$ different from $k$ the corresponding $\beta^{\prime}$ is different from $\beta$.

Inductively for every $\alpha \in \mathcal{A}$ we define $g_{\alpha}^{1}$ and $g_{\alpha}^{2}$ such that:

(1) For every $\alpha \in \mathcal{A}, \operatorname{supp} g_{\alpha}^{1}, \operatorname{supp} g_{\alpha}^{2} \subset D_{\alpha}$.

(2) If $w\left(f_{\alpha}\right)=m_{j_{0}}$, then $g_{\alpha}^{1}=e_{k_{\alpha}}^{*}$, where $\left|b_{k_{\alpha}}\right|=\max _{k \in D_{\alpha}}\left|b_{k}\right|$, and $g_{\alpha}^{2}=\varepsilon \sum_{k \in D_{\alpha}} e_{k}^{*}$.

(3) If $w\left(f_{\alpha}\right) \neq m_{j_{0}}$, then $g_{\alpha}^{1}=h_{\alpha}$ or $g_{\alpha}^{1}=e_{k_{\alpha}}^{*}+h_{\alpha}$, where $k_{\alpha} \notin \operatorname{supp} h_{\alpha}$, $h_{\alpha} \in W$ and $w\left(h_{\alpha}\right)=w\left(f_{\alpha}\right)$.

(4) For every $\alpha \in \mathcal{A}$,

$$
\left|f_{\alpha}\left(\sum_{k \in D_{\alpha}} b_{k} x_{k}\right)\right| \leq C\left(g_{\alpha}^{1}+g_{\alpha}^{2}\right)\left(\sum_{k \in D_{\alpha}}\left|b_{k}\right| e_{k}\right) .
$$


For every terminal node we set $g_{\alpha}^{1}=g_{\alpha}^{2}=0$ if $D_{\alpha}=\emptyset$, otherwise $g_{\alpha}^{1}=e_{k}^{*}$ if $D_{\alpha}=\{k\}$ and $g_{\alpha}^{2}=0$. Assume that we have defined the functionals $g_{\beta}^{1}$ and $g_{\beta}^{2}$ satisfying (1)-(4) for every $\beta \in \mathcal{A}$ with $|\beta|=k$, and let $\alpha \in \mathcal{A}$ with $|\alpha|=k-1$. If $D_{\alpha}=\emptyset$ we set $g_{\alpha}^{1}=g_{\alpha}^{2}=0$. Let $D_{\alpha} \neq \emptyset$. We distinguish two cases.

CASE 1. $w\left(f_{\alpha}\right)=m_{j} \neq m_{j_{0}}$.

Let $T_{\alpha}=D_{\alpha} \backslash \bigcup_{\beta \in S_{\alpha}} D_{\beta}=\left\{k: \alpha \in A_{k}\right\}$. We set $T_{\alpha}^{2}=\left\{k \in T_{\alpha}: m_{j_{k+1}}\right.$ $\left.\leq m_{j}\right\}$ and $T_{\alpha}^{1}=T_{\alpha} \backslash T_{\alpha}^{2}$. In the pointwise estimations we shall make below, we shall discard the coefficient $\lambda_{f_{2 i}}$, which appears in the definition of the special functionals, since $\left|\lambda_{f_{2 i}}\right| \leq 1$.

From condition (b) in the statement, it follows that for each $k \in T_{\alpha}^{2}$,

$$
\left|f_{\alpha}\left(x_{k}\right)\right| \leq \#\left(\operatorname{supp} x_{k}\right)\left\|f_{\alpha}\right\|_{\infty} \leq \#\left(\operatorname{supp} x_{k}\right) \frac{1}{m_{j}} \leq \varepsilon \leq C \varepsilon .
$$

We define

$$
g_{\alpha}^{2}=\varepsilon \sum_{k \in T_{\alpha}^{2}} e_{k}^{*}+\sum_{\beta \in S_{\alpha}} g_{\beta}^{2} .
$$

We observe that $\left\|g_{\alpha}^{2}\right\|_{\infty} \leq \varepsilon$, and that $\left|f_{\alpha}\left(x_{k}\right)\right| \leq C \varepsilon=C g_{\alpha}^{2}\left(e_{k}\right)$ for every $k \in T_{\alpha}^{2}$.

Let $T_{\alpha}^{1}=\left\{k_{1}<\ldots<k_{l}\right\}$. By the definition of $T_{\alpha}^{1}$ we have $m_{j}<m_{j_{k_{2}}}<$ $m_{j_{k_{3}}}<\ldots<m_{j_{k_{l}}}$. Thus condition (c) in the statement implies that

$$
\left|f_{\alpha}\left(x_{k_{i}}\right)\right| \leq \frac{C}{m_{j}}=\frac{1}{m_{j}} e_{k_{i}}^{*}\left(C e_{k_{i}}\right) \quad \text { for every } 2 \leq i \leq l .
$$

We set

$$
g_{\alpha}^{1}=e_{k_{1}}^{*}+\frac{1}{m_{j}}\left(\sum_{i=2}^{l} e_{k_{i}}^{*}+\sum_{\beta \in S_{\alpha}} g_{\beta}^{1}\right) .
$$

(The term $e_{k_{1}}^{*}$ does not appear if $w\left(f_{\alpha}\right)<m_{j_{k}}$ for every $k \in T_{\alpha}$.) We have to show that

$$
h_{\alpha}=\frac{1}{m_{j}}\left(\sum_{i=2}^{l} e_{k_{i}}^{*}+\sum_{\beta \in S_{\alpha}} g_{\beta}^{1}\right) \in W .
$$

From the inductive hypothesis, we have $g_{\beta}^{1}=h_{\beta}$ or $g_{\beta}^{1}=e_{k_{\beta}}^{*}+h_{\beta}, h_{\beta} \in W$, for every $\beta \in S_{\alpha}$. For $\beta \in S_{\alpha}$ such that $g_{\beta}^{1}=e_{k_{\beta}}^{*}+h_{\beta}$, let $E_{\beta}^{1}=\{n \in \mathbb{N}$ : $\left.n<k_{\beta}\right\}$ and $E_{\beta}^{2}=\left\{n \in \mathbb{N}: n>k_{\beta}\right\}$. We set $h_{\beta}^{1}=E_{\beta}^{1} h_{\beta}, h_{\beta}^{2}=E_{\beta}^{2} h_{\beta}$. For every $\beta$ such that $g_{\beta}^{1}=e_{k_{\beta}}^{*}+h_{\beta}$, the functionals $h_{\beta}^{1}, e_{k_{\beta}}^{*}, h_{\beta}^{2}$ are successive belonging to $W$, and for $\beta \neq \beta^{\prime} \in S_{\alpha}$ the corresponding functionals have disjoint ranges, since $\operatorname{supp} g_{\beta}^{1}$ is an interval (remark (iii) after the definition of $D_{\alpha}$ ). From remark (iv) after the definition of $D_{\alpha}$ we have $\# T_{\alpha}^{1} \leq n_{j}$. It 
follows that

$$
\begin{aligned}
\#\left(\{ e _ { k _ { i } } ^ { * } : 2 \leq i \leq l \} \cup \left\{e_{k_{\beta}}^{*}, h_{\beta}^{1}, h_{\beta}^{2}: \beta\right.\right. & \left.\in S_{\alpha}, g_{\beta}=e_{k_{\beta}}^{*}+h_{\beta}\right\} \\
& \left.\cup\left\{h_{\beta}: \beta \in S_{\alpha}, g_{\beta}=h_{\beta}\right\}\right) \leq 4 n_{j} .
\end{aligned}
$$

Therefore $h_{\alpha}=m_{j}^{-1}\left(\sum_{i=2}^{l} e_{k_{l}}^{*}+\sum_{\beta \in S_{\alpha}} g_{\beta}^{1}\right) \in W$.

It remains to show property (4). By (4.9) we have $\left|f_{\alpha}\left(x_{k_{i}}\right)\right| \leq C g_{\alpha}^{1}\left(e_{k_{i}}\right)$ for every $2 \leq i \leq l$, while

$$
\left|f_{\alpha}\left(x_{k_{1}}\right)\right| \leq\left\|x_{k_{1}}\right\| \leq C e_{k_{1}}^{*}\left(e_{k_{1}}\right)=g_{\alpha}^{1}\left(C e_{k_{1}}\right) .
$$

We also have

$$
\begin{aligned}
\left|f_{\alpha}\left(\sum_{k \in \bigcup_{\beta \in S_{\alpha}} D_{\beta}} b_{k} x_{k}\right)\right| & \leq \sum_{\beta \in S_{\alpha}}\left|f_{\alpha}\left(\sum_{k \in D_{\beta}} b_{k} x_{k}\right)\right| \\
& \leq \frac{1}{m_{j}} \sum_{\beta \in S_{\alpha}}\left|f_{\beta}\left(\sum_{k \in D_{\beta}} b_{k} x_{k}\right)\right| \\
& \leq \frac{1}{m_{j}} \sum_{\beta \in S_{\alpha}}\left(g_{\beta}^{1}+g_{\beta}^{2}\right)\left(C \sum_{k \in D_{\beta}}\left|b_{k}\right| e_{k}\right) \\
& \leq\left(g_{\alpha}^{1}+g_{\alpha}^{2}\right)\left(C \sum_{k \in D_{\alpha}}\left|b_{k}\right| e_{k}\right) .
\end{aligned}
$$

CASE 2. $w\left(f_{\alpha}\right)=m_{j_{0}}$.

In this case $D_{\alpha}$ is an interval of positive integers and $D_{\gamma}=\emptyset$ for every $\gamma \succ \alpha$. Let $k_{\alpha}$ be such that $b_{k_{\alpha}}=\max _{k \in D_{\alpha}}\left|b_{k}\right|$. We set

$$
g_{\alpha}^{1}=e_{k_{\alpha}}^{*}, \quad g_{\alpha}^{2}=\varepsilon \sum_{k \in D_{\alpha}} e_{k}^{*} .
$$

Then

$$
\begin{aligned}
\left|f_{\alpha}\left(\sum_{k \in D_{\alpha}} b_{k} x_{k}\right)\right| & \leq C\left(\max _{k \in D_{\alpha}}\left|b_{k}\right|+\varepsilon \sum_{k \in D_{\alpha}}\left|b_{k}\right|\right) \\
& =\left(g_{\alpha}^{1}+g_{\alpha}^{2}\right)\left(C \sum_{k \in D_{\alpha}}\left|b_{k}\right| e_{k}\right) .
\end{aligned}
$$

Definition 4.4. Let $k \in \mathbb{N}$. A vector $x \in c_{00}$ is said to be a $C$ - $\ell_{1}^{k}$ average if there exist $x_{1}<\ldots<x_{k}$ with $\left\|x_{i}\right\| \leq C\|x\|$ and $x=k^{-1} \sum_{i=1}^{k} x_{i}$. Moreover, if $\|x\|=1$ then $x$ is called a normalized $C$ - $\ell_{1}^{k}$ average.

LEMmA 4.5. Let $j \geq 1$ and $x$ a $C-\ell_{1}^{n_{j}}$ average. Then for every $n \leq n_{j-1}$ and every $E_{1}<\ldots<E_{n}$, we have

$$
\sum_{i=1}^{n}\left\|E_{i} x\right\| \leq C\left(1+\frac{2 n}{n_{j}}\right)<\frac{3}{2} C .
$$

We refer to [S] or [GM, Lemma 4] for a proof. 
Proposition 4.6. For every normalized block sequence $\left(y_{l}\right)_{l}$ and every $k \geq m_{2}$ there exists a linear combination of $\left(y_{l}\right)_{l}$ which is a normalized $2-\ell_{1}^{k}$ average.

Proof. Given $k \geq m_{2}$ there exists $j \in \mathbb{N}$ such that $m_{2 j-1}<k \leq m_{2 j+1}$. Recall that $n_{2 j+2}=\left(4 n_{2 j+1}\right)^{s_{2 j+1}}$ and $m_{2 j+2}^{3}<2^{s_{2 j+1}}$. Hence setting $s=$ $s_{2 j+1}$ we have $k^{s} \leq n_{2 j+2}$ and $2^{-s}<1 / m_{2 j+2}$. Observe that

$$
\left\|\sum_{i=1}^{k^{s}} y_{i}\right\| \geq \frac{k^{s}}{m_{2 j+2}} .
$$

Assuming that there is no normalized $2-\ell_{1}^{k}$ average in $\left\langle y_{i}: i \leq k^{s}\right\rangle$ and following the proof of Lemma 3 in $[\mathrm{GM}]$ we obtain

$$
\left\|\sum_{i=1}^{k^{s}} y_{i}\right\|<k^{s} \cdot 2^{-s} .
$$

Since $2^{-s}<1 / m_{2 j+2},(4.10)$ and (4.11) yield a contradiction.

Definition 4.7. A block sequence $\left(x_{k}\right)$ in $X_{\text {ius }}$ is said to be a $(C, \varepsilon)$ rapidly increasing sequence (R.I.S.) if there exists a strictly increasing sequence $\left(j_{k}\right)$ of positive integers such that:

(a) $\left\|x_{k}\right\| \leq C$.

(b) \#(range $\left.\left(x_{k}\right)\right) / m_{j_{k+1}}<\varepsilon$.

(c) $\left|f\left(x_{k}\right)\right| \leq C / w(f)$ for all $k=1,2, \ldots$ and $f \in K$ with $w(f)<m_{j_{k}}$.

REMARK 4.8. Let $\left(x_{k}\right)_{k}$ be a block sequence in $X_{\text {ius }}$ such that each $x_{k}$ is a normalized $2 C / 3-\ell_{1}^{n_{j_{k}}}$ average and let $\varepsilon>0$ be such that for each $k$, \# (range $\left.\left(x_{k}\right)\right)\left(1 / m_{j_{k+1}}\right)<\varepsilon$. Then Lemma 4.5 implies that condition (c) in the above definition is also satisfied and hence $\left(x_{k}\right)_{k}$ is a $(C, \varepsilon)$ R.I.S. In this case we shall call $\left(x_{k}\right)_{k}$ a $(C, \varepsilon)$ R.I.S. of $\ell_{1}$ averages. Observe also that Proposition 4.6 ensures that for every block sequence $\left(y_{l}\right)_{l}$ and every $\varepsilon>0$ there exists $\left(x_{k}\right)_{k}$ which is a $(3, \varepsilon)$ R.I.S. of $\ell_{1}$ averages. Then:

Proposition 4.9. Let $\left(x_{k}\right)_{i=1}^{n_{j}}$ be a $(C, \varepsilon)$ R.I.S. such that $\varepsilon \leq 1 / n_{j}$.

(1) For every $f \in K$,

$$
\left|f\left(\frac{1}{n_{j}} \sum_{k=1}^{n_{j}} x_{k}\right)\right| \leq \begin{cases}\frac{3 C}{m_{j} w(f)} & \text { if } w(f)<m_{j} \\ \frac{C}{w(f)}+\frac{2 C}{n_{j}} & \text { if } w(f) \geq m_{j} .\end{cases}
$$

In particular $\left\|n_{j}^{-1} \sum_{k=1}^{n_{j}} x_{k}\right\| \leq 2 C / m_{j}$. 
(2) If for $j_{0}=j$ assumption (d) of the basic inequality is satisfied (Proposition 4.3) for a linear combination $n_{j}^{-1} \sum_{i=1}^{n_{j}} b_{i} x_{i}$, where $\left|b_{i}\right| \leq 1$, then

$$
\left\|\frac{1}{n_{j}} \sum_{i=1}^{n_{j}} b_{i} x_{i}\right\| \leq \frac{4 C}{m_{j}^{3}} .
$$

(3) If $\left(x_{i}\right)_{i=1}^{n_{2 j}}$ is a $(3, \varepsilon)$ rapidly increasing sequence of $\ell_{1}$ averages then

$$
\frac{1}{m_{2 j}} \leq\left\|\frac{1}{n_{2 j}} \sum_{i=1}^{n_{2 j}} x_{i}\right\| \leq \frac{6}{m_{2 j}}
$$

Proof. The proof of (1) is an application of the basic inequality and Lemma 4.2. Indeed, for $f \in K$, the basic inequality implies that there exist $h_{1} \in W$ with $w(f)=w\left(h_{1}\right), t \in \mathbb{N}$ with $t \notin \operatorname{supp} h_{1}$, and $h_{2} \in c_{00}$ with $\left\|h_{2}\right\|_{\infty} \leq \varepsilon$, such that

$$
\left|f\left(\frac{1}{n_{j}} \sum_{k=1}^{n_{j}} x_{k}\right)\right| \leq\left(e_{t}^{*}+h_{1}+h_{2}\right) C\left(\frac{1}{n_{j}} \sum_{k=1}^{n_{j}} e_{k}\right) .
$$

Using Lemma 4.2 and the fact that $\varepsilon \leq 1 / n_{j}$ we obtain

$$
\begin{aligned}
\left|f\left(\frac{1}{n_{j}} \sum_{k=1}^{n_{j}} x_{k}\right)\right| \\
\quad \leq\left\{\begin{array}{l}
\frac{C}{n_{j}}+\frac{2 C}{w(f) m_{j}}+C \varepsilon \leq \frac{3 C}{w(f) m_{j}} \quad \text { if } w(f)<m_{j}, \\
\frac{C}{n_{j}}+\frac{C}{w(f)}+C \varepsilon \leq \frac{C}{w(f)}+\frac{2 C}{n_{j}} \quad \text { if } w(f) \geq m_{j} .
\end{array}\right.
\end{aligned}
$$

To prove (2) we observe that the basic inequality yields the existence of $h_{1}$, $h_{2}$ such that $h_{1}$ has a tree $\left(h_{\alpha}\right)_{\alpha \in \mathcal{A}}$ such that $w\left(h_{\alpha}\right) \neq m_{j}$ for every $\alpha \in \mathcal{A}$ and $\left\|h_{2}\right\|_{\infty} \leq \varepsilon$. This and Lemma 4.2 yield

$$
\begin{aligned}
\left|f\left(\frac{1}{n_{j}} \sum_{k=1}^{n_{j}} b_{k} x_{k}\right)\right| & \leq\left(e_{t}^{*}+h_{1}+h_{2}\right) C\left(\frac{1}{n_{j}} \sum_{k=1}^{n_{j}} e_{k}\right) \\
& \leq \frac{C}{n_{j}}+\frac{2 C}{m_{j}^{3}}+C \varepsilon \leq \frac{4 C}{m_{j}^{3}} .
\end{aligned}
$$

The upper estimate in (3) follows from (1) for $C=3$. For the lower estimate in (3), for every $i \leq n_{2 j}$ we choose a functional $f_{i}$ belonging to the pointwise closure of $K$ such that $f_{i}\left(x_{i}\right)=1$ and range $\left(f_{i}\right) \subset \operatorname{range}\left(x_{i}\right)$. Then it is easy to see that the functional $f=m_{2 j}^{-1} \sum_{i=1}^{n_{2 j}} f_{i}$ belongs to the same set and provides the required result.

Proposition 4.10. The space $X_{\mathrm{ius}}$ is reflexive. 
Proof. As explained after the definition of the norming set $K$, the basis is boundedly complete. Therefore to show that $X_{\text {ius }}$ is reflexive we need to prove that the basis is shrinking.

Assume the contrary, i.e. there exists $x^{*}=w^{*}-\sum_{n=1}^{\infty} b_{n} e_{n}^{*}$ and $x^{*} \notin \overline{\left\langle e_{n}^{*}\right\rangle}$. Then there exists $\varepsilon>0$ and successive intervals $\left(E_{k}\right)_{k}$ such that $\left\|E_{k} x^{*}\right\|>\varepsilon$. Choose $\left(x_{k}\right)_{k}$ in $X_{\text {ius }}$ such that $\operatorname{supp} x_{k} \subset E_{k},\left\|x_{k}\right\|=1$ and $x^{*}\left(x_{k}\right)>\varepsilon$. It follows that every convex combination $\sum a_{k} x_{k}$ satisfies

$$
\left\|\sum a_{k} x_{k}\right\|>\varepsilon \text {. }
$$

Next for $j$ sufficiently large such that $4 / \varepsilon m_{2 j}<\varepsilon$ we define $y_{1}, \ldots, y_{n_{2 j}}$ to be a $\left(2 / \varepsilon, 1 / n_{2 j}\right)$ R.I.S. of $\ell_{1}$ averages such that each $y_{i}$ is some average of $\left(x_{k}\right)_{k}$. Proposition $4.9(1)$ yields

$$
\left\|\frac{1}{n_{2 j}}\left(y_{1}+\ldots+y_{n_{2 j}}\right)\right\| \leq \frac{4}{m_{2 j} \varepsilon}<\varepsilon .
$$

Clearly (4.17) contradicts (4.16) and the basis is shrinking.

The structure of $\mathcal{B}\left(X_{\text {ius }}\right)$

Definition 4.11. A sequence $\chi=\left(x_{1}, f_{1}, \ldots, x_{n_{2 j+1}}, f_{n_{2 j+1}}\right)$ is said to be a dependent sequence of length $n_{2 j+1}$ if the following conditions are satisfied:

(i) There exists a special sequence

$$
\phi=\left(x_{1}, f_{1}, y_{2}, f_{2}, \ldots, x_{2 i-1}, f_{2 i-1}, y_{2 i}, f_{2 i}, \ldots, y_{n_{2 j+1}}, f_{n_{2 j+1}}\right)
$$

of length $n_{2 j+1}$ such that $\operatorname{supp} y_{2 i}=\operatorname{supp} x_{2 i}$ and $\left\|y_{2 i}-x_{2 i}\right\| \leq 1 / n_{j_{2 i}}^{2}$, where $j_{i+1}=\sigma\left(\phi_{i}\right)$ for $1 \leq i<n_{2 j+1}$.

(ii) For $i \leq n_{2 j+1} / 2$ we have

$$
x_{2 i}=\frac{c_{2 i}}{n_{j_{2 i}}} \sum_{l=1}^{n_{j_{2 i}}} x_{l}^{2 i},
$$

where $\left(x_{l}^{2 i}\right)_{l}$ is a $\left(3,1 / n_{j_{2 i}}\right)$ R.I.S. of $\ell_{1}$ averages and $c_{2 i} \in(0,1)$.

(iii) $f_{2 i}\left(x_{2 i}\right) \geq 1 / 12 m_{j_{2 i}}$.

The following is a consequence of the previous results, and we sketch the proof of it.

Lemma 4.12. Let $\left(y_{k}\right)_{k}$ be a normalized block sequence in $X_{\text {ius }}$ and $\left(e_{n}\right)_{n \in M}$ be a subsequence of its basis. Then for all $j \in \mathbb{N}$ there exists a dependent sequence

$$
\chi=\left(x_{1}, f_{1}, \ldots, x_{n_{2 j+1}}, f_{n_{2 j+1}}\right)
$$

of length $n_{2 j+1}$ such that for each $i \leq n_{2 j+1} / 2, x_{2 i-1} \in\left\langle e_{n}\right\rangle_{M}$ and $x_{2 i}$ $\in\left\langle y_{k}\right\rangle_{k}$. 
Proof. Let $j_{1} \in \mathbb{N}$ be even such that $m_{j_{1}}^{1 / 2}>n_{2 j+1}$. We set

$$
x_{1}=\frac{1}{n_{j_{1}}} \sum_{i=1}^{n_{j_{1}}} e_{1, i}, \quad f_{1}=\frac{1}{m_{j_{1}}} \sum_{i=1}^{n_{j_{1}}} e_{1, i}^{*}
$$

such that $x_{1} \in\left\langle e_{n}\right\rangle_{M}$. Let $j_{2}=\sigma\left(x_{1}, f_{1}\right)$. Using Proposition 4.6 we choose a $\left(3,1 / n_{j_{2}}\right)$ R.I.S. $\left(x_{l}^{2}\right)_{l=1}^{n_{j_{2}}} \subset\left\langle y_{k}\right\rangle_{k}$ such that $x_{1}<x_{l}^{2}$ for every $l \leq n_{j_{2}}$. Next for every $l \leq n_{j_{2}}$ we choose a functional $f_{l}^{2} \in K$ such that $f_{l}^{2}\left(x_{l}^{2}\right) \geq \frac{2}{3}\left\|x_{l}^{2}\right\| \geq \frac{2}{3}$ and range $\left(f_{l}^{2}\right) \subset \operatorname{range}\left(x_{l}^{2}\right)$. We set

$$
f_{2}=\frac{1}{m_{j_{2}}} \sum_{l=1}^{n_{j_{2}}} f_{l}^{2}, \quad x_{2}=\frac{c_{2}}{n_{j_{2}}} \sum_{l=1}^{n_{j_{2}}} x_{l}^{2}, \quad \text { where } \quad c_{2}=\frac{1}{6}\left(1-\frac{m_{j_{2}}}{n_{j_{2}}^{2}}\right) .
$$

From Proposition 4.9 , it follows that $\left\|x_{2}\right\| \leq 1 / m_{j_{2}}-1 / n_{j_{2}}^{2}$. We also have

$$
f_{2}\left(x_{2}\right) \geq \frac{1}{m_{j_{2}}} \frac{c_{2}}{n_{j_{2}}} \sum_{l=1}^{n_{j_{2}}} f_{l}^{2}\left(x_{l}^{2}\right) \geq \frac{2}{3} \frac{c_{2}}{m_{j_{2}}} \geq \frac{1}{12 m_{j_{2}}}
$$

We choose $y_{2} \in \mathbf{Q}$ (that is, $y_{2}$ is a finite sequence with rational coordinates) such that $\left\|y_{2}-x_{2}\right\| \leq 1 / n_{j_{2}}^{2}$ and $\operatorname{supp} y_{2}=\operatorname{supp} x_{2}$. It follows that $\left\|y_{2}\right\| \leq$ $1 / m_{j_{2}}$ and therefore $\left(x_{1}, f_{1}, y_{2}, f_{2}\right)$ is a special sequence of length 2 .

We set $j_{3}=\sigma\left(x_{1}, f_{1}, y_{2}, f_{2}\right)$ and we choose

$$
x_{3}=\frac{1}{n_{j_{3}}} \sum_{l=1}^{n_{j_{3}}} e_{3, l}, \quad f_{3}=\frac{1}{m_{j_{3}}} \sum_{l=1}^{n_{j_{3}}} e_{3, l}^{*}
$$

such that range $\left(y_{2}\right) \cup \operatorname{range}\left(f_{2}\right)<\operatorname{range}\left(x_{3}\right)$ and $x_{3} \in\left\langle e_{n}\right\rangle_{M}$. Next we choose $x_{4}, f_{4}$ and $y_{4}$ as in the second step; it is clear that the procedure goes through up to the choice of $x_{n_{2 j+1}}, f_{n_{2 j+1}}$ and $y_{n_{2 j+1}}$.

REMARK 4.13. (a) Observe that the proof of Lemma 4.12 shows that if $\chi=\left(x_{1}, f_{1}, \ldots, x_{n_{2 j+1}}, f_{n_{2 j+1}}\right)$ is a dependent sequence, then for every $i \leq n_{2 j+1} / 2$ we have

$$
x_{2 i}=\frac{c_{2 i}}{n_{j_{2 i}}} \sum_{l=1}^{n_{j_{2 i}}} x_{l}^{2 i}
$$

where $\left(x_{l}^{2 i}\right)_{l}$ is a $\left(3, n_{j_{2 i}}\right)$ R.I.S., $j_{2 i}=\sigma\left(\phi_{2 i-1}\right)$ and $c_{2 i} \leq 1 / 6$. It follows from Proposition 4.9 that $\left\|m_{j_{2 i}} x_{2 i}\right\| \leq 1$, and also if $f \in K$ and $w(f)<m_{j_{2 i}}$ then $f\left(m_{j_{2 i}} x_{2 i}\right) \leq 2 / w(f)$.

(b) Definition 4.11 essentially implies that a dependent sequence is a small perturbation of a special sequence. Its necessity occurs from the restriction in the definition of the special sequence $\phi=\left(x_{1}, f_{1}, \ldots, x_{n_{2 j+1}}, f_{n_{2 j+1}}\right)$ that each $x_{i} \in \mathbf{Q}$ (i.e. $x_{i}(n)$ is a rational number), not permitting to find such elements $x_{i}$ in every block subspace. 
Next we state the basic estimates of averages related to dependent sequences.

Lemma 4.14. Let $\chi=\left(x_{1}, f_{1}, \ldots, x_{n_{2 j+1}}, f_{n_{2 j+1}}\right)$ be a dependent sequence of length $n_{2 j+1}$. Then

$$
\left\|\frac{1}{n_{2 j+1}} \sum_{i=1}^{n_{2 j+1}}(-1)^{i+1} m_{j_{i}} x_{i}\right\| \leq \frac{8}{m_{2 j+1}^{3}}
$$

where $m_{j_{i}}=w\left(f_{i}\right)$.

Lemma 4.15. Let $\phi=\left(x_{1}, f_{1}, \ldots, x_{n_{2 j+1}}, f_{n_{2 j+1}}\right)$ be a special sequence. For every $i \leq n_{2 j+1} / 2$, let $\sigma\left(x_{1}, f_{1}, \ldots, x_{2 i-1}, f_{2 i-1}\right)=j_{2 i}$ and let

$$
y_{2 i}=\frac{m_{j_{2 i}}}{n_{j_{2 i}}} \sum_{l=1}^{n_{j_{2 i}}} e_{k_{l}}
$$

be such that

$$
\operatorname{supp} f_{2 i} \cap \operatorname{supp} y_{2 i}=\emptyset, \quad \operatorname{supp} f_{2 i-1}<\operatorname{supp} y_{2 i}<\operatorname{supp} f_{2 i+1} .
$$

Then

$$
\left\|\frac{1}{n_{2 j+1}} \sum_{i=1}^{n_{2 j+1} / 2} y_{2 i}\right\| \leq \frac{8}{m_{2 j+1}^{3}} .
$$

These two lemmas are the key ingredients for proving the main results on the structure of $X_{\text {ius }}$ and $\mathcal{B}\left(X_{\text {ius }}\right)$. We proceed with the proof of the main results; the proof of the two lemmas will be given at the end.

Proposition 4.16. Let $M \in[\mathbb{N}]$ and let $\left(y_{k}\right)_{k}$ be a normalized block sequence. Then

$$
\operatorname{dist}\left(S_{\left\langle e_{n}\right\rangle_{M}}, S_{\left\langle y_{k}\right\rangle_{k}}\right)=0
$$

Proof. For a given $\varepsilon>0$ we choose $j \in \mathbb{N}$ such that $8 / m_{2 j+1}^{2}<\varepsilon$. From Lemma 4.12 there exists a dependent sequence $\chi=\left(x_{1}, f_{1}, \ldots, x_{n_{2 j+1}}, f_{n_{2 j+1}}\right)$ such that $x_{2 i-1} \in\left\langle e_{n}\right\rangle_{M}, x_{2 i} \in\left\langle y_{k}\right\rangle_{k}$ for every $i \leq n_{2 j+1} / 2$. Set

$$
e=\frac{m_{2 j+1}}{n_{2 j+1}} \sum_{i=1}^{n_{2 j+1} / 2} m_{j_{2 i-1}} x_{2 i-1}, \quad y=\frac{m_{2 j+1}}{n_{2 j+1}} \sum_{i=1}^{n_{2 j+1} / 2} m_{j_{2 i}} x_{2 i} .
$$

Then $e \in\left\langle e_{n}: n \in M\right\rangle$ and $y \in\left\langle y_{i}: i \in M\right\rangle$. From Lemma 4.14 we have $\|e-y\| \leq 8 / m_{2 j+1}^{2}$. To obtain a lower estimate of the norm of $e$ and $y$ we consider the functional

$$
f=\frac{1}{m_{2 j+1}} \sum_{i=1}^{n_{2 j+1} / 2} \lambda_{f_{2 i}} f_{2 i-1}+f_{2 i}
$$

where $\lambda_{f_{2 i}}=f_{2 i}\left(m_{j_{2 i}} y_{2 i}\right)$ and $\phi=\left(x_{1}, f_{1}, y_{2}, f_{2}, \ldots, y_{n_{2 j+1}}, f_{n_{2 j+1}}\right)$ is the special sequence associated to the dependent sequence $\chi$. From the definition 
of the dependent sequence, $f_{2 i}\left(m_{j_{2 i}} x_{2 i}\right) \geq 1 / 12$, and $\left\|x_{2 i}-y_{2 i}\right\| \leq 1 / n_{j_{2 i}}^{2}$ for every $i \leq n_{2 j+1} / 2$. It follows that

$$
\lambda_{f_{2 i}}=f\left(m_{j_{2 i}} y_{2 i}\right) \geq f\left(m_{j_{2 i}} x_{2 i}\right)-m_{j_{2 i}}\left\|x_{2 i}-y_{2 i}\right\|>\frac{1}{12}-\frac{1}{m_{j_{2 i}}^{2}}>\frac{1}{24} .
$$

Therefore

$$
\begin{aligned}
& \|e\| \geq f(e)=\frac{m_{2 j+1}}{m_{2 j+1}} \sum_{i=1}^{n_{2 j+1} / 2} \frac{\lambda_{f_{2 i}} f_{2 i-1}\left(m_{j_{2 i-1}} x_{2 i-1}\right)}{n_{2 j+1}} \geq \frac{1}{48} \\
& \|y\| \geq f(y)=\frac{m_{2 j+1}}{m_{2 j+1}} \sum_{i=1}^{n_{2 j+1} / 2} \frac{f_{2 i}\left(m_{j_{2 i}} x_{2 i}\right)}{n_{2 j+1}} \geq \frac{1}{24} .
\end{aligned}
$$

These lower estimates and the fact that $\|e-y\| \leq 8 / m_{2 j+1}^{2}$ easily yield the desired result.

Lemma 4.17. Let $T: X_{\text {ius }} \rightarrow X_{\text {ius }}$ be a bounded operator. Then

$$
\lim _{n} \operatorname{dist}\left(T e_{n}, \mathbb{R} e_{n}\right)=0 \text {. }
$$

Proof. Without loss of generality we may assume that $\|T\|=1$. Since $\left(e_{n}\right)$ is weakly null, by a small perturbation of $T$ we may assume that $T e_{n}$ is a finite block, $T e_{n} \in \mathbf{Q}$ and $\min \operatorname{supp} T e_{n} \rightarrow \infty$ as $n \rightarrow \infty$. Let $I\left(e_{n}\right)$ be the smallest interval containing $\operatorname{supp} T e_{n} \cup \operatorname{supp} e_{n}$. Passing to a subsequence $\left(e_{n}\right)_{n \in M}$, we may assume that $I\left(e_{n}\right)<I\left(e_{m}\right)$ for all $n, m \in M$ with $n<m$.

If the result is not true, we may assume, on passing to a further subsequence, that there exists $\delta>0$ such that

$$
\operatorname{dist}\left(T e_{n}, \mathbb{R} e_{n}\right)>2 \delta \quad \text { for every } n \in M \text {. }
$$

It follows that $\left\|P_{n-1} T e_{n}\right\|>\delta$ or $\left\|\left(I-P_{n}\right) T e_{n}\right\|>\delta$. Therefore for every $n \in M$ we can choose $x_{n}^{*} \in K$ such that

$$
x_{n}^{*}\left(T e_{n}\right) \geq \delta, \quad \operatorname{range}\left(x_{n}^{*}\right) \cap \operatorname{range}\left(e_{n}\right)=\emptyset, \quad \operatorname{range}\left(x_{n}^{*}\right) \subset I\left(e_{n}\right) .
$$

Since $T$ is bounded, for every $j \in \mathbb{N}$ we have

$$
\left\|T\left(\frac{1}{n_{2 j}} \sum_{i=1}^{n_{2 j}} e_{k_{i}}\right)\right\| \leq\|T\|\left\|\frac{1}{n_{2 j}} \sum_{i=1}^{n_{2 j}} e_{k_{i}}\right\|=\frac{1}{m_{2 j}} .
$$

Also for every $j \in \mathbb{N}$ and $k_{1}<\ldots<k_{n_{2 j}}$ in $M$, the functional $h_{2 j}=$ $m_{2 j}^{-1} \sum_{i=1}^{n_{2 j}} x_{k_{i}}^{*}$ is in $K$ and

$$
\left\|T\left(\frac{1}{n_{2 j}} \sum_{i=1}^{n_{2 j}} e_{k_{i}}\right)\right\|=\left\|\frac{1}{n_{2 j}} \sum_{i=1}^{n_{2 j}} T e_{k_{i}}\right\| \geq h_{2 j}\left(\frac{1}{n_{2 j}} \sum_{i=1}^{n_{2 j}} T e_{k_{i}}\right) \geq \frac{\delta}{m_{2 j}} .
$$


We now consider a special sequence $\phi=\left(x_{1}, f_{1}, \ldots, x_{n_{2 j+1}}, f_{n_{2 j+1}}\right)$ which is defined as follows: for every $i \geq 0$,

$$
\begin{aligned}
x_{2 i+1} & =\frac{1}{n_{\sigma\left(\phi_{2 i}\right)}} \sum_{j=1}^{n_{\sigma\left(\phi_{2 i}\right)}} e_{2 i+1, j}, \quad f_{2 i+1}=\frac{1}{m_{\sigma\left(\phi_{2 i}\right)}} \sum_{j=1}^{n_{\sigma\left(\phi_{2 i}\right)}} e_{2 i+1, j}^{*}, \\
x_{2 i} & =\frac{1}{n_{\sigma\left(\phi_{2 i-1}\right)}} \sum_{j=1}^{n_{\sigma\left(\phi_{2 i-1}\right)}} T e_{2 i, j}, \quad f_{2 i}=\frac{1}{m_{\sigma\left(\phi_{2 i-1}\right)}} \sum_{j=1}^{n_{\sigma\left(\phi_{2 i-1}\right)}} x_{2 i, j}^{*},
\end{aligned}
$$

where $e_{i, l} \in\left\{e_{n}: n \in M\right\}, x_{2 i, j}^{*}, T e_{2 i, j}$ satisfies (4.21), and $I\left(e_{i, l}\right)<I\left(e_{s, j}\right)$ if either $i<s$, or $i=s$ and $l<j$. This is possible by our assumption $I\left(e_{n}\right)<I\left(e_{m}\right)$ for $n, m \in M$ with $n<m$. Observe that $f_{2 i}\left(m_{\sigma\left(\phi_{2 i-1}\right)} x_{2 i}\right) \geq \delta$ and also that range $\left(f_{l}\right) \cap \operatorname{range}\left(x_{2 i}\right)=\emptyset$ for every $l \neq 2 i$. Consider now the following vector:

$$
x=\frac{1}{n_{2 j+1}} \sum_{i=1}^{n_{2 j+1} / 2} \frac{m_{\sigma\left(\phi_{2 i-1}\right)}}{n_{\sigma\left(\phi_{2 i-1}\right)}} \sum_{j=1}^{n_{\sigma\left(\phi_{2 i-1}\right)}} e_{2 i, j} .
$$

Then

$$
T x=\frac{1}{n_{2 j+1}} \sum_{i=1}^{n_{2 j+1} / 2} m_{\sigma\left(\phi_{2 i-1}\right)} x_{2 i}
$$

and

$$
\|T x\| \geq \frac{1}{m_{2 j+1}} \sum_{i=1}^{n_{2 j+1} / 2}\left(\lambda_{f_{2 i}} f_{2 i-1}+f_{2 i}\right) T x \geq \frac{\delta}{2 m_{2 j+1}} .
$$

On the other hand, if

$$
y_{2 i}=\frac{m_{\sigma\left(\phi_{2 i-1}\right)}}{n_{\sigma\left(\phi_{2 i-1}\right)}} \sum_{j=1}^{n_{\sigma\left(\phi_{2 i-1}\right)}} e_{2 i, j}
$$

then supp $y_{2 i} \cap \operatorname{supp} f_{2 i}=\emptyset$ and $x_{2 i-1}<y_{2 i}<x_{2 i+1}$ for every $i \leq n_{2 j+1} / 2$, and therefore by Lemma 4.15 ,

$$
\|x\|=\left\|\frac{1}{n_{2 j+1}} \sum_{i=1}^{n_{2 j+1} / 2} y_{2 i}\right\| \leq \frac{8}{m_{2 j+1}^{3}} .
$$

Hence $\|T\| \geq \frac{\delta}{16} m_{2 j+1}^{2}$, a contradiction for $j$ sufficiently large.

Proposition 4.18. Let $T: X_{\text {ius }} \rightarrow X_{\text {ius }}$ be a bounded operator. Then there exists $\lambda \in \mathbb{R}$ such that $T-\lambda I$ is strictly singular.

Proof. By Lemma 4.17 there exist $\lambda \in \mathbb{R}$ and $M \in[\mathbb{N}]$ such that $\lim _{n \in M}\left\|T e_{n}-\lambda e_{n}\right\|=0$. Let $\varepsilon>0$. Passing to a further subsequence $\left(e_{n_{k}}\right)_{k}$, we may assume that $\left\|T e_{n_{k}}-\lambda e_{n_{k}}\right\| \leq \varepsilon 2^{-k}$ for every $k \in \mathbb{N}$. It 
follows that the restriction of $T-\lambda I$ to $\left[e_{n_{k}}, k \in \mathbb{N}\right]$ is of norm less than $\varepsilon$. By Proposition 4.16 it follows that $T-\lambda I$ is strictly singular.

The following two corollaries are consequences of Proposition 4.18 (see $[\mathrm{GM}])$.

Corollary 4.19. There is no nontrivial projection $P: X_{\mathrm{ius}} \rightarrow X_{\mathrm{ius}}$.

COROLlary 4.20. The space $X_{\text {ius }}$ is not isomorphic to any proper subspace of it.

It remains to prove Lemmas 4.14 and 4.15. We start with the following.

Lemma 4.21. Let $j \in \mathbb{N}$ and $n_{2 j+1}<m_{j_{1}}<m_{j_{2}}<\ldots<m_{j_{2 r}}$ be such that $2 r \leq n_{2 j+1}<m_{j_{1}}^{1 / 2}$. Let also $j_{0} \in \mathbb{N}$ be such that $m_{j_{0}} \neq m_{j_{i}}$ for every $i=1, \ldots, 2 r$ and $m_{j_{0}}^{1 / 2}>n_{2 j+1}$. Then if $h_{1}<\ldots<h_{2 r} \in K$ are such that $w\left(h_{i}\right)=m_{j_{i}}$ for every $i=1, \ldots, 2 r$, then:

(a)

$$
\left|\left(\sum_{k=1}^{r} \lambda_{2 k-1} h_{2 k-1}+h_{2 k}\right)\left(\frac{m_{j_{0}}}{n_{j_{0}}} \sum_{l=1}^{n_{j_{0}}} e_{k_{l}}\right)\right|<\frac{1}{n_{2 j+1}}
$$

for any real numbers $\left(\lambda_{2 k-1}\right)_{k=1}^{r}$ with $\left|\lambda_{2 k-1}\right| \leq 1$ for every $k \leq r$.

(b) If $\left(x_{l}\right)_{l=1}^{n_{j_{0}}}$ is a $\left(3,1 / n_{j_{0}}\right)$ R.I.S. of $\ell_{1}$ averages, then

$$
\left|\left(\sum_{k=1}^{r} \lambda_{2 k-1} h_{2 k-1}+h_{2 k}\right)\left(\frac{m_{j_{0}}}{n_{j_{0}}} \sum_{l=1}^{n_{j_{0}}} x_{l}\right)\right| \leq \frac{1}{n_{2 j+1}}
$$

for any real numbers $\left(\lambda_{2 k-1}\right)_{k=1}^{r}$ with $\left|\lambda_{2 k-1}\right| \leq 1$ for every $k \leq r$.

Proof. We shall give the proof of (b) and we shall indicate the minor changes for the proof of (a).

From the estimates on the R.I.S. (Proposition 4.9), for every $k \leq 2 r$ we have

$$
\left|h_{k}\left(\frac{m_{j_{0}}}{n_{j_{0}}} \sum_{l=1}^{n_{j_{0}}} x_{l}\right)\right| \leq \begin{cases}9 / w\left(h_{k}\right) & \text { if } w\left(h_{k}\right)<m_{j_{0}}, \\ 3 / m_{r}+6 / n_{j_{0}} & \text { if } w\left(h_{k}\right)=m_{r}>m_{j_{0}} .\end{cases}
$$

Since $m_{j+1}=m_{j}^{5}$ for every $j$ and $\left|\lambda_{2 k-1}\right| \leq 1$ for every $k \leq r$, from (4.24) we get

$$
\begin{aligned}
& \left|\left(\sum_{k=1}^{r} \lambda_{2 k-1} h_{2 k-1}+h_{2 k}\right)\left(\frac{m_{j_{0}}}{n_{j_{0}}} \sum_{l=1}^{n_{j_{0}}} x_{l}\right)\right| \\
& \quad \leq \sum_{k: w\left(h_{k}\right)<m_{j_{0}}} \frac{9}{w\left(h_{k}\right)}+\sum_{r>j_{0}} \frac{3}{m_{r}}+\frac{12 r}{n_{j_{0}}} \leq \frac{10}{w\left(h_{1}\right)}+\frac{4}{m_{j_{0}}^{2}}+\frac{12 r}{n_{j_{0}}}<\frac{1}{n_{2 j+1}} .
\end{aligned}
$$

For the proof of (a), using Lemma 4.2 we get an inequality corresponding to (4.24), from which (4.22) follows. 
Proof of Lemma 4.14. Let $\chi=\left(x_{1}, f_{1}, \ldots, x_{n_{2 j+1}}, f_{n_{2 j+1}}\right)$ be a dependent sequence and $\phi=\left(y_{1}, f_{1}, y_{2}, f_{2}, \ldots, y_{n_{2 j+1}}, f_{n_{2 j+1}}\right)$ the special sequence associated to $\chi$. In the rest of the proof we shall assume that $\chi=\phi$. The general proof follows by slight and obvious modifications. Hence we assume that $\phi=\left(x_{1}, f_{1}, \ldots, x_{n_{2 j+1}}, f_{n_{2 j+1}}\right)$.

From Lemma 4.2 and Remark 4.13(a) it follows that the sequence $\left(m_{j_{i}} x_{i}\right)_{i=1}^{n_{2 j+1}}$ satisfies assumptions (a), (c) of the basic inequality for $C=2$. Furthermore the properties of the function $\sigma$ imply that assumption (b) is also satisfied for $\varepsilon=1 / n_{2 j+1}$.

The rest of the proof is devoted to establishing that the sequence $\left(m_{j_{i}} x_{i}\right)_{i}$ satisfies the crucial condition (d) for $m_{j_{0}}=m_{2 j+1}$ and $\left(b_{i}\right)_{i}=\left((-1)^{i+1} / n_{2 j+1}\right)_{i}$.

First we consider $f \in K_{\phi}$. Then $f$ is of the form

$$
f=E\left(\frac{\varepsilon}{m_{2 j+1}}\left(\lambda_{f_{2}^{\prime}} f_{1}+f_{2}^{\prime}+\ldots+\lambda_{f_{n_{2 j+1}}^{\prime}} f_{n_{2 j+1}-1}+f_{n_{2 j+1}}^{\prime}\right)\right),
$$

where $\varepsilon \in\{-1,1\}$ and $E$ is an interval of $\mathbb{N}$. Recall that $w\left(f_{2 i}^{\prime}\right)=w\left(f_{2 i}\right)$ and $\operatorname{supp} f_{2 i}^{\prime}=\operatorname{supp} f_{2 i}$ and therefore range $\left(f_{2 i}^{\prime}\right) \cap \operatorname{range}\left(x_{k}\right)=\emptyset$ for every $k \neq 2 i$. Let

$$
i_{0}=\min \left\{i \leq n_{2 j+1} / 2: \operatorname{supp} f \cap\left(\operatorname{range}\left(x_{2 i-1}\right) \cup \operatorname{range}\left(x_{2 i}\right)\right) \neq \emptyset\right\} .
$$

Then

$$
\begin{aligned}
\left|f\left(\sum_{i=1}^{n_{2 j+1}}(-1)^{i+1} m_{j_{i}} x_{i}\right)\right| & \\
= & \left|E \frac{1}{m_{2 j+1}} \sum_{k=1}^{n_{2 j+1} / 2}\left(\lambda_{f_{2 k}^{\prime}} f_{2 k-1}+f_{2 k}^{\prime}\right)\left(\sum_{i=1}^{n_{2 j+1}}(-1)^{i+1} m_{j_{i}} x_{i}\right)\right| \\
\leq & \frac{1}{m_{2 j+1}}\left|\lambda_{f_{2 i_{0}}^{\prime}} E f_{2 i_{0}-1}\left(m_{j_{2 i_{0}-1}} x_{2 i_{0}-1}\right)-E f_{2 i_{0}}^{\prime}\left(m_{j_{2 i}} x_{2 i_{0}}\right)\right| \\
& \quad+\frac{1}{m_{2 j+1}}\left|\sum_{i=i_{0}+1}^{n_{2 j+1} / 2}\left(\lambda_{f_{2 i}^{\prime}} f_{2 i-1}\left(m_{j_{2 i-1}} x_{2 i-1}\right)-f_{2 i}^{\prime}\left(m_{j_{2 i}} x_{2 i}\right)\right)\right| .
\end{aligned}
$$

To estimate the expressions in (4.25) and (4.26), we partition the set $\left\{i_{0}, \ldots, n_{2 j+1} / 2\right\}$ into $A=\left\{i: f_{2 i}^{\prime}\left(x_{2 i}\right) \neq 0\right\}$ and $B$. For every $i \in A$, $i>i_{0}$, since $\lambda_{f_{2 i}^{\prime}}=f_{2 i}^{\prime}\left(m_{j_{2 i}} x_{2 i}\right)$, we have

$$
\begin{aligned}
\lambda_{f_{2 i}^{\prime}} f_{2 i-1}\left(m_{j_{2 i-1}} x_{2 i-1}\right)-f_{2 i}^{\prime} & \left(m_{j_{2 i}} x_{2 i}\right) \\
& =f_{2 i}^{\prime}\left(m_{j_{2 i}} x_{2 i}\right)-f_{2 i}^{\prime}\left(m_{j_{2 i}} x_{2 i}\right)=0 .
\end{aligned}
$$


For every $i \in B$ we have $f_{2 i}^{\prime}\left(x_{2 i}\right)=0$, and therefore $\left|\lambda_{f_{2 i}^{\prime}}\right|=1 / n_{2 j+1}^{2}$ (see (2.6)). It follows that for every $i \in B$ with $i>i_{0}$,

$$
\left|\lambda_{f_{2 i}^{\prime}} f_{2 i-1}\left(m_{j_{2 i-1}} x_{2 i-1}\right)-f_{2 i}^{\prime}\left(m_{j_{2 i}} x_{2 i}\right)\right|=\left|\lambda_{f_{2 i}^{\prime}}\right|=\frac{1}{n_{2 j+1}^{2}} .
$$

For the term in (4.25), distinguishing whether or not $E f_{2 i_{0}-1}=0$ and whether $i_{0} \in A$ or $i_{0} \in B$, it follows easily using the previous arguments that

$$
\left|\lambda_{f_{2 i_{0}}^{\prime}} E f_{2 i_{0}-1}\left(m_{j_{2 i_{0}-1}} x_{2 i_{0}-1}\right)-E f_{2 i_{0}}^{\prime}\left(m_{j_{2 i_{0}}} x_{2 i_{0}}\right)\right| \leq 1
$$

Summing up (4.27)-(4.29) we have

$$
\begin{aligned}
& \left|f\left(\frac{1}{n_{2 j+1}} \sum_{i=1}^{n_{2 j+1}}(-1)^{i+1} m_{j_{i}} x_{i}\right)\right| \\
& \leq \frac{1}{m_{2 j+1}}\left(\frac{1}{n_{2 j+1}}+\frac{1}{n_{2 j+1}^{2}}\right)<\frac{1}{n_{2 j+1}} .
\end{aligned}
$$

Consider now a special sequence $\psi=\left(y_{1}, g_{1}, \ldots, y_{n_{2 j+1}}, g_{n_{2 j+1}}\right)$. Let $i_{1}=$ $\min \left\{i \in\left\{1, \ldots, n_{2 j+1}\right\}: y_{i} \neq x_{i}\right.$ or $\left.g_{i} \neq f_{i}\right\}$, and let $k_{0} \in \mathbb{N}$ be such that $i_{1}=2 k_{0}-1$ or $2 k_{0}$.

Consider a functional $g \in K_{\psi}$ which is defined from this special sequence. Then

$$
g=E\left(\frac{1}{m_{2 j+1}}\left(\lambda_{g_{2}^{\prime}} g_{1}+g_{2}^{\prime}+\ldots+\lambda_{g_{n_{2 j+1}}^{\prime}} g_{n_{2 j+1}-1}+g_{n_{2 j+1}}^{\prime}\right)\right),
$$

where $E$ is an interval of $\mathbb{N}$ and $w\left(g_{2 i}^{\prime}\right)=w\left(g_{2 i}\right)$ for every $i \leq n_{2 j+1} / 2$. Observe that range $\left(x_{i}\right) \cap \operatorname{range}\left(g_{k}\right)=\emptyset$ for every $i \geq i_{1}$ and every $k<i_{1}$. Let

$$
i_{0}=\min \left\{i \leq n_{2 j+1} / 2: \operatorname{supp} g \cap\left(\operatorname{range}\left(x_{2 i-1}\right) \cup \operatorname{range}\left(x_{2 i}\right)\right) \neq \emptyset\right\} .
$$

Let $i_{0}<k_{0}$. Then

$$
\left|g\left(\sum_{i=1}^{n_{2 j+1}}(-1)^{i+1} m_{j_{i}} x_{i}\right)\right|
$$

$$
\begin{aligned}
\leq & \frac{1}{m_{2 j+1}}\left(\left|E \lambda_{g_{2 i_{0}}^{\prime}} g_{2 i_{0}-1}\left(m_{j_{2 i_{0}-1}} x_{2 i_{0}-1}\right)-E g_{2 i_{0}}^{\prime}\left(m_{j_{2 i_{0}}} x_{2 i_{0}}\right)\right|\right. \\
& +\mid \sum_{i=i_{0}+1}^{k_{0}-1}\left(\lambda_{g_{2 i}^{\prime}} g_{2 i-1}\left(m_{j_{2 i-1}} x_{2 i-1}\right)-g_{2 i}^{\prime}\left(m_{j_{2 i}} x_{2 i}\right) \mid\right) \\
& +\frac{1}{m_{2 j+1}}\left|\sum_{k \geq k_{0}}\left(\lambda_{g_{2 k}^{\prime}} g_{2 k-1}+g_{2 k}^{\prime}\right)\left(\sum_{i \geq k_{0}} m_{j_{2 i-1}} x_{2 i-1}-m_{j_{2 i}} x_{2 i}\right)\right|,
\end{aligned}
$$

where the sum in (4.32) makes sense when $i_{0}<k_{0}-1$. If $i_{0} \geq k_{0}$ we get 


$$
\begin{aligned}
& \left|g\left(\sum_{i=1}^{n_{2 j+1}}(-1)^{i+1} m_{j_{i}} x_{i}\right)\right| \\
& \quad \leq \frac{1}{m_{2 j+1}}\left|E \sum_{k \geq k_{0}}\left(\lambda_{g_{2 k}^{\prime}} g_{2 k-1}+g_{2 k}^{\prime}\right)\left(\sum_{i \geq i_{0}} m_{j_{2 i-1}} x_{2 i-1}-m_{j_{2 i}} x_{2 i}\right)\right| .
\end{aligned}
$$

The proof of the upper estimate for the two cases is almost identical, so we shall give the proof in the case $i_{0}<k_{0}$.

As in the previous case, for the term in (4.31), (4.32) we have

$$
\begin{aligned}
& \left|E \lambda_{g_{2 i_{0}}^{\prime}} g_{2 i_{0}-1}\left(m_{j_{2 i_{0}-1}} x_{2 i_{0}-1}\right)-E g_{2 i_{0}}^{\prime}\left(m_{j_{2 i_{0}}} x_{2 i_{0}}\right)\right| \\
& +\mid \sum_{i=i_{0}+1}^{k_{0}-1}\left(\lambda_{g_{2 i}^{\prime}} g_{2 i-1}\left(m_{j_{2 i-1}} x_{2 i-1}\right)-g_{2 i}^{\prime}\left(m_{j_{2 i}} x_{2 i}\right) \mid \leq 2 .\right.
\end{aligned}
$$

To estimate the sum in (4.33), first we observe that from the injectivity of $\sigma$ it follows that there exists at most one $k \geq i_{1}$ such that

$$
w\left(g_{k}\right) \in\left\{m_{j_{i}}: i_{1} \leq i \leq n_{2 j+1}\right\} .
$$

Let $2 i-1 \geq i_{1}$ be such that $m_{j_{2 i-1}} \neq w\left(g_{k}\right)$ for every $k \geq i_{1}$. Then the functionals $g_{2 k-1}, g_{2 k}^{\prime}, k \geq k_{0}$, satisfy the assumptions of Lemma 4.21, and therefore

$$
\left|\sum_{k \geq k_{0}}\left(\lambda_{g_{2 k}^{\prime}} g_{2 k-1}+g_{2 k}^{\prime}\right)\left(m_{j_{2 i-1}} x_{2 i-1}\right)\right| \leq \frac{1}{n_{2 j+1}} .
$$

Also for every $2 i \geq i_{1}$ such that $m_{j_{2 i}} \neq w\left(g_{k}\right)$ for every $k \geq i_{1}$, the functionals $g_{2 k-1}, g_{2 k}^{\prime}, k \geq k_{0}$, satisfy the assumptions of Lemma 4.21 , and so

$$
\left|\sum_{k \geq k_{0}}\left(\lambda_{g_{2 k}^{\prime}} g_{2 k-1}+g_{2 k}^{\prime}\right)\left(m_{j_{2 i}} x_{2 i}\right)\right| \leq \frac{1}{n_{2 j+1}} .
$$

For the unique $i \geq i_{1}$ such that there exists $k \geq i_{1}$ with $w\left(g_{k}\right)=m_{j_{i}}$ (if such an $i$ exists), we have, using Lemma 4.21,

$$
\left|\sum_{k \geq k_{0}}\left(\lambda_{g_{2 k}^{\prime}} g_{2 k-1}+g_{2 k}^{\prime}\right)\left(m_{j_{i}} x_{i}\right)\right| \leq 1+\frac{1}{n_{2 j+1}} .
$$

Now we distinguish the cases of $i_{1}=2 k_{0}-1$ and $i_{1}=2 k_{0}$. If $i_{1}=2 k_{0}-1$, we have range $\left(g_{k}\right) \cap \operatorname{range}\left(x_{i}\right)=\emptyset$ for every $k<2 k_{0}-1$ and every $i \geq 2 k_{0}-1$, and from (4.35)-(4.37) we get

$$
\begin{aligned}
\mid \sum_{k \geq k_{0}}\left(\lambda_{g_{2 k}^{\prime}} g_{2 k-1}+g_{2 k}^{\prime}\right) & \left(\frac{1}{n_{2 j+1}} \sum_{i=2 k_{0}-1}^{n_{2 j+1}}(-1)^{i+1} m_{j_{i}} x_{i}\right) \mid \\
\leq & \frac{1}{n_{2 j+1}}\left(1+\frac{1}{n_{2 j+1}}+\frac{n_{2 j+1}}{n_{2 j+1}}\right)<\frac{3}{n_{2 j+1}} .
\end{aligned}
$$


If $i_{1}=2 k_{0}$ then range $\left(x_{2 k_{0}-1}\right) \cap \operatorname{range}\left(g_{k}\right)=\emptyset$ for every $k \geq 2 k_{0}$ and $k<2 k_{0}-1$, and from (4.35)-(4.37) we get

$$
\begin{aligned}
\left|\sum_{k \geq k_{0}}\left(\lambda_{g_{2 k}^{\prime}} g_{2 k-1}+g_{2 k}^{\prime}\right)\left(\frac{1}{n_{2 j+1}} \sum_{i=2 k_{0}-1}^{n_{2 j+1}}(-1)^{i+1} m_{j_{i}} x_{i}\right)\right| \\
\leq \frac{1}{n_{2 j+1}}\left(\left|\lambda_{g_{2 k_{0}-1}^{\prime}} g_{2 k_{0}-1}\left(m_{j_{2 k_{0}-1}} x_{2 k_{0}-1}\right)\right|\right. \\
\left.+\left|\sum_{k \geq k_{0}}\left(\lambda_{g_{2 k}^{\prime}} g_{2 k-1}+g_{2 k}^{\prime}\right)\left(\sum_{i=2 k_{0}}^{n_{2 j+1}}(-1)^{i+1} m_{j_{i}} x_{i}\right)\right|\right) \\
\leq \frac{1}{n_{2 j+1}}+\frac{1}{n_{2 j+1}}\left(1+\frac{1}{n_{2 j+1}}+\frac{n_{2 j+1}}{n_{2 j+1}}\right)<\frac{4}{n_{2 j+1}} .
\end{aligned}
$$

From (4.34), (4.38) and (4.39) we get

$$
\left|g\left(\frac{1}{n_{2 j+1}} \sum_{i=1}^{n_{2 j+1}}(-1)^{i+1} m_{j_{i}} x_{i}\right)\right| \leq \frac{1}{m_{2 j+1}}\left(\frac{2}{n_{2 j+1}}+\frac{4}{n_{2 j+1}}\right)<\frac{1}{n_{2 j+1}} .
$$

The inequalities (4.30) and (4.40) show that indeed condition (d) is satisfied for $\varepsilon=1 / n_{2 j+1}$. Proposition 4.9(2) now yields the desired result.

Proof of Lemma 4.15. We shall follow similar arguments to those in the proof of Lemma 4.14. We shall establish conditions (a)-(d) of the basic inequality for $C=2, \varepsilon=1 / n_{2 j+1}$ and $m_{j_{0}}=m_{2 j+1}$. Lemma 4.2 shows that the sequence $\left(y_{2 i}\right)_{i}$ satisfies (a) and (c) for $C=2$. Furthermore the properties of the function $\sigma$ imply that (b) is also satisfied for $\varepsilon=1 / n_{2 j+1}$.

To establish condition (d) we shall show that for every $f \in K$ with $w(f)=m_{2 j+1}$,

$$
\left|f\left(\frac{1}{n_{2 j+1}} \sum_{i=1}^{n_{2 j+1} / 2} y_{2 i}\right)\right| \leq \frac{1}{m_{2 j+1}}\left(\frac{1}{n_{2 j+1}}+\frac{1}{n_{2 j+1}}\right)<\frac{1}{n_{2 j+1}} .
$$

First observe that for every $f \in K_{\phi}$ of the form

$$
f=E \frac{1}{m_{2 j+1}} \sum_{k=1}^{n_{2 j+1} / 2}\left(\lambda_{f_{2 k}^{\prime}} f_{2 k-1}+f_{2 k}^{\prime}\right)
$$

we have

$$
f\left(\frac{1}{n_{2 j+1}} \sum_{i=1}^{n_{2 j+1} / 2} y_{2 i}\right)=0 .
$$

This is due to $\operatorname{supp} f_{2 i}^{\prime}=\operatorname{supp} f_{2 i}$ and $\operatorname{supp} f_{2 i-1}<y_{2 i}<\operatorname{supp} f_{2 i+1}$ for every $i \leq n_{2 j+1} / 2$. 
Let $\phi=\left(z_{1}, g_{1}, z_{2}, g_{2}, \ldots, z_{n_{2 j+1}}, g_{n_{2 j+1}}\right)$ be a special sequence of length $n_{2 j+1}$ and let

$$
f=E \frac{1}{m_{2 j+1}} \sum_{k=1}^{n_{2 j+1} / 2}\left(\lambda_{g_{2 k}^{\prime}} g_{2 k-1}+g_{2 k}^{\prime}\right) \in K_{\phi}
$$

We may assume that $E=\mathbb{N}$. Let $i_{1}=\min \left\{i \leq n_{2 j+1}: z_{i} \neq x_{i}\right.$ or $\left.f_{i} \neq g_{i}\right\}$, and let $k_{0} \in \mathbb{N}$ be such that $i_{1}=2 k_{0}-1$ or $i_{1}=2 k_{0}$. Observe that range $\left(g_{k}\right) \cap$ range $\left(y_{2 i}\right)=\emptyset$ for every $k<i_{1}$ and every $2 i \geq i_{1}$.

From the injectivity of $\sigma$, it follows that there exists at most one $k \geq i_{1}$ such that

$$
w\left(g_{k}\right) \in\left\{m_{j_{i}}: i_{1} \leq i \leq n_{2 j+1}\right\} .
$$

Let $2 i \geq i_{1}$ be such that $w\left(g_{k}\right) \neq m_{j_{2 i}}$ for all $k \geq i_{1}$. Then the functionals $g_{2 k-1}, g_{2 k}^{\prime}, k \geq k_{0}$, satisfy the assumptions of Lemma 4.21(a), and therefore

$$
\left|\left(\sum_{k \geq k_{0}} \lambda_{g_{2 k}^{\prime}} g_{2 k-1}+g_{2 k}^{\prime}\right)\left(y_{2 i}\right)\right|<\frac{1}{n_{2 j+1}} .
$$

For the unique $2 i \geq i_{1}$ such that there exists $k \geq i_{1}$ with $w\left(g_{k}\right)=m_{j_{2 i}}$ (if such a $2 i$ exists), we have

$$
\left|\left(\sum_{k \geq k_{0}} \lambda_{g_{2 k}^{\prime}} g_{2 k-1}+g_{2 k}^{\prime}\right)\left(y_{2 i}\right)\right|<1+\frac{1}{n_{2 j+1}} .
$$

Summing up (4.41)-(4.42) we get

$$
\left|f\left(\frac{1}{n_{2 j+1}} \sum_{i=1}^{n_{2 j+1} / 2} y_{2 i}\right)\right| \leq \frac{1}{m_{2 j+1}}\left(\frac{1}{n_{2 j+1}}+\frac{1}{n_{2 j+1}}\right)<\frac{1}{n_{2 j+1}}
$$

Inequality (4.43) implies that condition (d) of the basic inequality is satisfied, and Proposition 4.9 yields the desired result.

Remark 4.22. As pointed out by A. Pełczyński, there is no obstacle to the existence of an indecomposable closed subspace of a Banach space with an unconditional basis. However our space is not such an example. In particular the space $X_{\text {ius }}$ does not embed into a Banach space with an unconditional f.d.d. This follows from the property that no subsequence of the basis $\left(e_{n}\right)_{n}$ is an unconditional basic sequence. For the same reason the space $X_{\text {ius }}$ is not a quotient of a space with a shrinking unconditional f.d.d. [O].

\section{References}

[AD] S. A. Argyros and I. Deliyanni, Examples of asymptotic $\ell_{1}$ Banach spaces, Trans. Amer. Math. Soc. 349 (1997), 973-995.

[AF] S. A. Argyros and V. Felouzis, Interpolating hereditarily indecomposable Banach spaces, J. Amer. Math. Soc. 13 (2000), 243-294. 
[AT1] S. A. Argyros and A. Tolias, Methods in the theory of hereditarily indecomposable Banach spaces, Mem. Amer. Math. Soc., to appear.

[AT2] - - - Indecomposability and unconditionality in duality, Geom. Funct. Anal., to appear.

[BD] J. Bernues and I. Deliyanni, Families of finite subsets of $\mathbb{N}$ of low complexity and Tsirelson type spaces, Math. Nachr. 222 (2001), 15-29.

[G] W. T. Gowers, A solution to Banach's hyperplane problem, Bull. London Math. Soc. 26 (1994), 523-530.

[G2] -, Ramsey methods in Banach spaces, in: Handbook of the Geometry of Banach Spaces, Vol. 2, W. B. Johnson and J. Lindenstrauss, (eds.), Elsevier, Amsterdam, 2003.

[G3] -, An infinite Ramsey theorem and some Banach-space dichotomies, Ann. of Math. (2) 156 (2002), 797-833.

[GM] W. T. Gowers and B. Maurey, The unconditional basic sequence problem, J. Amer. Math. Soc. 6 (1993), 851-874.

[LT] J. Lindenstrauss and L. Tzafriri, Classical Banach Spaces I, Springer, 1977.

[O] E. Odell, On quotients of Banach spaces having shrinking unconditional bases, Illinois J. Math. 36 (1992), 681-695.

[S] Th. Schlumprecht, An arbitrarily distortable Banach space, Israel J. Math. 76 (1991), 81-95.

Department of Mathematics

National Technical University of Athens

Athens, Greece

E-mail: sargyros@math.ntua.gr
Department of Sciences

Section of Mathematics Technical University of Crete

Chania, Crete, Greece E-mail: amanouss@science.tuc.gr

Received May 21, 2002

Revised version July 4, 2003 